TAO, Vol. 11, No. 1, 21-52, March 2000

\title{
COSMIC System Description
}

\author{
Christian Rocken ${ }^{1}{ }^{*}$, Ying-Hwa Kuo ${ }^{2}$, William S. Schreiner ${ }^{1}$, Doug Hunt ${ }^{1}$, \\ Sergey Sokolovskiy ${ }^{1}$ and Chris McCormick ${ }^{3}$
}

(Manuscript received 18 May 1999, in final form 8 October 1999)

\begin{abstract}
The Constellation Observing System for Meteorology Ionosphere and Climate (COSMIC) is a satellite mission for the Earth sciences that will make use of recent developments in remote sensing, communications technology, and computing to solve some of the most important geo-scientific issues today. COSMIC plans to launch eight Low Earth Orbit (LEO) satellites in 2003. Each of these spacecraft will carry three science payloads for weather and space weather research and prediction, climate monitoring, and geodesy: 1) GPS occultation receiver, 2) Tiny Ionospheric Photometer (TIP), and 3) Triband Beacon transmitters (TBB). Each of the LEOs will track the GPS satellites as they are occulted behind the Earth limb to retrieve up to $\mathbf{5 0 0}$ daily profiles of key ionospheric and atmospheric properties. Every day the constellation will provide globally about 4000 GPS soundings. TIP will measure electron densities at the peak of the F2 layer along the satellite track. TBB transmissions will be received on the ground for high-resolution tomographic reconstruction of the ionospheric electron density. COSMIC continuos precise tracking of all GPS satellites in view, also promise to benefit geodetic studies. The COSMIC system includes the LEO satellites, ground data reception and spacecraft control stations, data analysis centers and the data communications networks. This paper gives a COSMIC science overview and describes the COSMIC system.
\end{abstract}

(Key words: Global Positioning System (GPS), GPS-Meteorology

(GPS-MET), Remote sensing, Atmospheric sounding systems,

Radio occultation, Space Weather, Gravity, COSMIC

(Constellation Observing System for Meteorology, Ionosphere and Climate)

\footnotetext{
${ }^{1}$ GPS Science and Technology Program, UCAR, USA

${ }^{2}$ Mesoscale and Microscale Meteorology Division, National Center for Atmospheric Research, UCAR, USA

${ }^{3}$ Broad Reach Inc., Boulder, CO USA

* Corresponding author address: Dr. Christian Rocken, UCAR, 3300 Mitchell Lane, Boulder, CO 80301 USA; E-mail: rocken@ucar.edu
} 


\section{COSMIC SCIENCE AND PRODUCTS}

\subsection{Science Mission for COSMIC - Overview}

Data from the Constellation Observing System for Meteorology Ionosphere and Climate (COSMIC) will shed a new and revealing light on a wide variety of natural phenomena that are of considerable importance to the scientific community and to society as a whole. COSMIC is an interdisciplinary satellite mission that will address some of the most intriguing questions in the Earth sciences today.

In meteorology the COSMIC data set will allow us to investigate the global water vapor distribution and map the atmospheric flow of water vapor that is so crucial for weather analysis and prediction (Crook, 1996, Anthes et al., 1997, Kuo et al., 1997). The high vertical resolution of the data set (Karayel and Hinson, 1997, Gurbunov and Gurvitch, 1998) will provide accurate geopotential heights (Leroy, 1997), enable the detection of gravity waves from the upper troposphere to the stratosphere (Tsuda et al., 2000), reveal the height and shape of the tropopause globally with unprecentented accuracy, support the investigation of fronts and other baroclinic structures (Kuo et al., 1998), and improve our understanding of tropopause-stratosphere exchange processes. One key goal of COSMIC is to demonstrate improvements in the performance of numerical weather models, especially in polar and oceanic re-gions.

For climate studies COSMIC will monitor Earth's atmosphere with unprecedented longterm stability, resolution, coverage, and accuracy. It will thus collect a data set for the detection of climate variability and change, the separation of natural and anthropogenic causes, and the testing of climate models (Yuan et al., 1993, North and Stevens, 1998, Stevens, 1998, Leroy, 1998). Upper-tropospheric refractivity data from COSMIC may shed new light on the recent controversy over the role that tropical convection plays in climate feedback (Lindzen, 1990, Rind, 1998). COSMIC will enhance studies of changes associated with El Nino events especially in remote oceanic regions, and it will enable scientists to monitor the response of the global atmosphere to regional events such as large volcanic eruptions, the Kuwait oil fires, or the large Indonesian forest fires.

In the ionosphere COSMIC data will accelerate the development of physical models for space weather prediction by providing dense, accurate, and global electron density measurements for model testing and initialization (Hajj et al., 1994, Howe et al., 1998, Rius et al., 1997, 1998). The large volume of high quality ionospheric observations from COSMIC will significantly advance space weather research. Scientists will be able to observe the response of the global ionosphere to the impact of a solar storm as its effects propagate around the globe. New revelations from this data set will improve physical ionospheric models and thus contribute to the development of predictive skills for space weather.

Each satellite in the COSMIC constellation will be tracked with high accuracy using GPS. This orbit trajectory information shall be used to improve our knowledge of the Earth's gravity field and geoid (Lemoine, 1998). Improvements in the gravity field have far reaching impact on the Earth Sciences and on operational civilian and military applications. Changes in the gravity field reveal changes in the Earth's mass distribution due to core-mantel, tectonic, 
hydrological, glacial, oceanographic, or atmospheric effects. Better gravity fields also lead to more accurate satellite orbit estimation and can thus improve GPS surveying and benefit geodetic science.

\subsection{COSMIC Data Products}

Atmospheric and ionospheric profiles are derived by the radio occultation technique. As a signal travels through the atmosphere it is retarded and bent. This results in a phase and Doppler shift, which can be measured very accurately by the GPS receiver aboard the low-Earth orbiting COSMIC satellites. Since the transmitter and receiver positions and velocities are accurately know from precise orbit determination (POD), signal bending, $\alpha$, as a function of impact parameter, $\boldsymbol{a}$, (see Fig. 1) can be computed from the Doppler shift observed at the Low Earth Orbiter (LEO). From the basic bending angle vs. impact parameter data, vertical profiles of refractivity as a function of tangent point radius, $r$, can be derived. Further analysis converts refractivity to electron density in the ionosphere (Hajj and Romans, 1998, Schreiner et al., 1999). In the neutral atmosphere (stratosphere and troposphere), the bending angle-derived refractivity profiles, are primarily a function of temperature, pressure, and water vapor (i.e. Gurvitch and Sokolovskiy 1983, Melbourne et al., 1994, Feng et al., 1995, Ware et al., 1996, Hoeg et al., 1996, Hocke, 1997, Kursinski et al., 1996, 1997, Rocken et al., 1997). Effects due to hydrometeors and other particulates are generally ignored (Solheim et al., 1999).

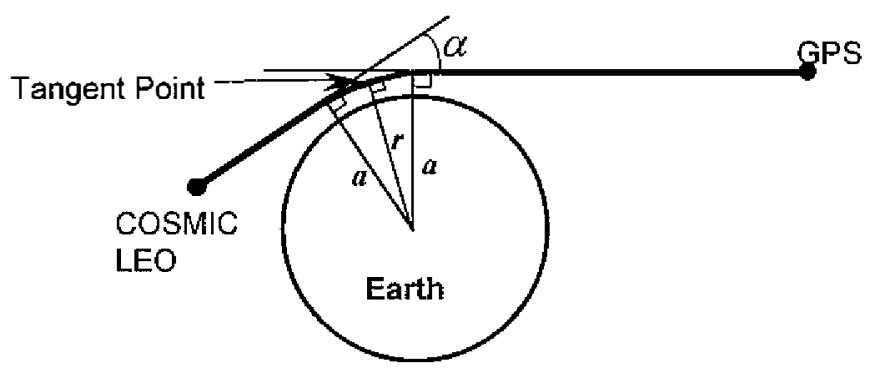

Fig. 1. Occultation observation geometry.

Where water vapor is negligible, such as in the stratosphere and upper troposphere, pressure and temperature may be obtained directly from the refractivity. Where water vapor is present in significant amounts, it is impossible, without additional independent data, to separate out the effects of temperature and water vapor. However, if a reasonably accurate independent estimate of temperature is available (for example, from a global analysis or a 12-hour forecast), accurate profiles of water vapor and total pressure can be obtained (Fig. 2).

Eyre (1994) stated that the most powerful use of COSMIC neutral atmosphere data is to assimilate the refractivity or bending angle profiles directly into numerical models. In this way a minimum number of assumptions are made. Numerical experiments have shown that assimilation of refractivity (Zou et al., 1995) and bending angles directly into numerical models causes the model's temperature, water vapor, and wind fields to adjust toward actual atmospheric values (Kuo et al., 1997, Zou et al., 1999, 2000). 

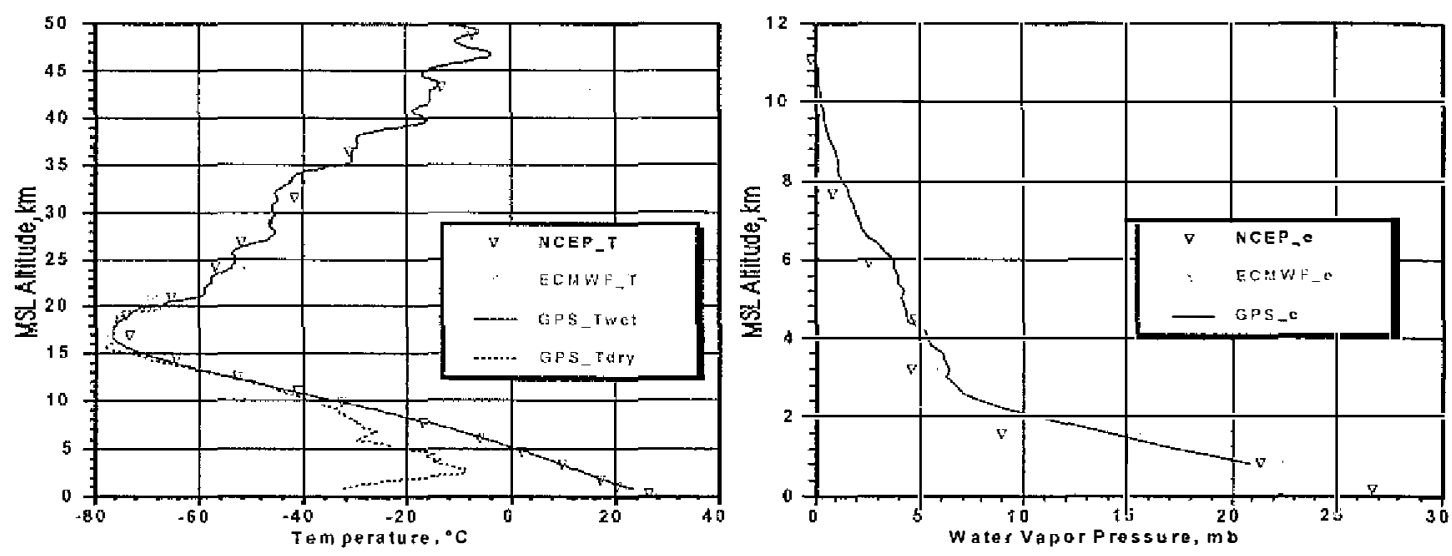

Fig. 2. GPS/MET occultation profiles from 11:40 UTC on June 22, 1995, at $9^{\circ} \mathrm{S}$ $172^{\circ} \mathrm{W}$ compared with global analysis. GPS/MET temperature (left) and water vapor (right) are compared to NCEP and ECMF model values. Water vapor in this example is measured to $\sim 800 \mathrm{~m}$ above the surface (from Rocken et al., 1997).

Photometric measurements from the Tiny Ionospheric Photometer (TIP) instrument will be used in conjunction with the ionospheric GPS data. In particular TIP will provide horizontal gradients in electron density at the peak of the F2 layer, along the satellite orbit track. TIP will measure the naturally occuring nighttime emission of neutral oxygen at $135.6 \mathrm{~nm}$. This ernission is produced by the recombination of $\mathrm{O}^{+}$ions and electrons and is proportional to the square of the electron density in the ionospheric $F$ region. Since horizontal gradients of electron density are a limiting error source for occultation inversions in the ionosphere (Hajj and Romans, 1998, Schreiner et al., 1999), combined analysis of TIP and GPS data promises improved retrievals of nighttime ionospheric profiles.

Phase data transmissions from the COSMIC Tri-band-beacon (TBB) instrument at 150, 400 and $1067 \mathrm{MHz}$ can be received at ground stations worldwide. These data will be converted to line-of-sight total electron content (TEC) observations that can be processed with 2dimensional ionospheric tomography techniques (Bust et al., 1994; Bernhardt et al., 1998). TBB data can also be combined with the other ionospheric observations in tomographic and physical data assimilation models to compute global four-dimensional electron density fields (Hajj et al., 1994, Howe et al., 1998, Rius et al., 1997, 1998). While meteorological data assimilation techniques are already quite advanced, data assimilation in the ionosphere is still in its infancy. However, recent programs sponsored by the Department of Defense promise to improve the situation within the next few years, and COSMIC data will complement these model development efforts.

COSMIC data will also be used for monitoring ionospheric scintillation. Scintillation is caused by small scale irregularities in the ionospheric $F$ layer predominantly between 225-400 $\mathrm{km}$. Scintillation is weak at mid-latitudes but can be strong enough to cause serious communication outages in the auroral and especially equatorial post-sunset regions. COSMIC plans to 
compute scintillation parameters such as the S4 index (standard deviation of received power divided by the mean value) on-orbit, and to transmit these values via the TBB data link to the global network of ground beacon receiver stations. TBB phase and amplitude observations at ground receiver sites will provide additional scintillation observations at VHF (150 MHz), UHF (400 MHz) and L-band (1067MHz). These near real-time data will be useful for generating communication outage maps and, in conjunction with the COSMIC GPS and TIP observations, for understanding and prediction of conditions leading to scintillation.

The COSMIC magnetometer, while primarily a navigation instrument, could also provide valuable data for space weather research. This would require some modifications to the current mission design, which are still under discussion. The COSMIC satellite vector magnetometers will register variations due to electric currents flowing along geomagnetic-field lines in the auroral regions (field-aligned currents), generally at magnetic latitudes of 60-75 degrees, but extending to lower magnetic latitudes during magnetic storms (Richmond and Kamide, 1998). Horizontal deflections are of the order of hundreds of nanotesla (nT), often exceeding $1000 \mathrm{nT}$ during disturbed periods. In addition to the use of the vector deflections, additional information can be obtained simply from looking at the power of the magnetic fluctuations at roughly $1-10 \mathrm{~Hz}$ (Brian Anderson, private communication, 1999). Such fluctuations correlate well with passage through field-aligned currents, and can thus be used to determine the size of the auroral oval, an important parameter for space weather purposes. COSMIC satellites at 72 degree inclination will pass through the auroral ovals of both hemispheres on most orbits.

In addition to the occultation GPS data, COSMIC GPS receivers will collect $0.1 \mathrm{~Hz}$ dual frequency phase data from all GPS satellites in view. Gravity field and GPS orbit improvement will be achieved by ingesting the COSMIC GPS phase observations and other relevant information about the satellite (i.e., mass istribution, attitude) into well established geodetic and orbit parameter estimation models. This will have a direct impact on geodetic science by improving gravity field models, the reference frame, and GPS satellite orbits, and an indirect impact through improved global fields of pressure, temperature, moisture, and electron density. Accurate long term monitoring of the gravity field will improve the spatial and temporal understanding of a variety of mass motion processes within the solid Earth, atmosphere, ocean, and cryosphere. The current state-of-the-art gravity model is the Earth Gravity Model EGM96 (Lemoine et al., 1998). For COSMIC data to provide significant improvement over EGM96, it is necessary for pairs of satellites to fly in tandem (with $300-1000 \mathrm{~km}$ separation) at the lowest altitude possible. E. Pavlis (1998, unpublished results) computed a gravity field with 32 days of simulated COSMIC data. His results show an expected order of magnitude improvement in coefficient uncertainty over EGM96, out to degree and order 20-40 (spatial resolution of 1000-500 km). Geoid errors also show order of magnitude improvement over EGM96 out to at least degree and order 40. Such improvements to the static gravity field and geoid will benefit many past and future geodetic satellite missions. Improvements to estimates of temporal variations of the gravity field coefficients will not be obtainable with COSMIC because of the necessity to stay at low altitudes for a long time. The COSMIC engineering teams at UCAR and at Taiwan's National Space Program Office (NSPO) are designing the mission to maximize the value of gravity observations. This requires a trade-off between the scientific demand for low orbits for as long as possible and the engineering requirement to 
minimize the required on-board propellant.

In summary, COSMIC aims to satisfy the following high-level science requirements.

Table 1. COSMIC Science Requirements.

\begin{tabular}{|c|c|c|}
\hline Data Parameter & Science Requirement & Comment \\
\hline Vertical GPS occ. resolution & $0.3-1.5 \mathrm{~km}$ & $0.3 \mathrm{~km}$ near surface, $1.5 \mathrm{~km}$ at $45 \mathrm{~km}$ \\
\hline Horizontal GPS occ. resolution & $300-600 \mathrm{~km}$ & Smaller for fronts with large slope \\
\hline Bending angle profile & $1.510^{-6} \mathrm{rad}$ & $\begin{array}{l}\text { Limited mainly by residual ionospheric error } \\
\text { and data noise }\end{array}$ \\
\hline Refractivity profile & $<1 \%$ & As good as $0.2 \%$ between $10-30 \mathrm{~km}$ \\
\hline Temperature profile & $1 \mathrm{deg} C$ & In 0-40 km height range, assuming dry air \\
\hline Water vapor profile & $<1-10 \%$ & $\begin{array}{l}\text { Between } 0-5 \mathrm{~km} \text {, assuming model temp. error } \\
\text { of } 1-2 \text { deg; errors between } 5-10 \mathrm{~km} \text { larger }\end{array}$ \\
\hline Geopotential height vs. pressure & $10-20 \mathrm{~m}$ & Between $0-30 \mathrm{~km}$ (worse at solar maximum) \\
\hline Electron density profile & $<1-20 \%$ & $\begin{array}{l}\text { Profile quality near the F2 peak- can be worse } \\
\text { elsewhere; Limited by horizontal gradients }\end{array}$ \\
\hline GPS TEC & $0.001 \mathrm{TECU}$ & Relative TEC (absolute $\sim 3-5$ TECU) \\
\hline TBB TEC & $0.003 \mathrm{TECU}$ & Relative TEC (absolute 1-3 TECU) \\
\hline TIP peak foF2 density, $\mathrm{NmF2}$ & $<1-10 \%$ & $\begin{array}{l}\text { Performs better at high electron densities } \\
\text { Works only on the night side of the globe }\end{array}$ \\
\hline Magnetometer measurement * & 10 nano-tesla (nt) & $\begin{array}{l}\text { Precision@1 } 1 \mathrm{~Hz} \text { rate, } 500 \mathrm{nt} \text { accuracy } \\
\text { (*easibility still under consideration) }\end{array}$ \\
\hline Scintillation GPS & $\mathrm{n} / \mathrm{a}$ & $100 \mathrm{~Hz}$ SNR data used to determine $S 4$ \\
\hline Scintillation TBB & $\mathrm{n} / \mathrm{a}$ & $50-1000 \mathrm{~Hz}$ SNR data \\
\hline LEO Position & $\sim 10 \mathrm{~cm}$ & 3-D rms position error (not critical) \\
\hline LEO velocity & $<0.1 \mathrm{~mm} / \mathrm{s}$ & $\begin{array}{l}\text { Relative velocity error for paired satellites } \\
\text { will be smaller due to common errors }\end{array}$ \\
\hline
\end{tabular}

Many of the science requirements in Table 1 are based on experience from GPS/MET (i.e., Rocken et al., 1997) and theoretical error studies (i.e., Kursinski et al., 1997). The ionospheric profiling errors with the wide range of $1-20 \%$ are based on recent $\mathrm{NmF} 2$ comparisons from GPS/MET (i.e., Schreiner et al., 1999) and do not apply in the E-layer where the agreement is often worse. The accuracy of these results is limited by the assumption of horizontal ionospheric homogeneity. Combined analysis of TIP, TBB and GPS data promises to improve the quality of retrieved ionospheric profiles. At this point funding for the conversion of the magnetometer from a navigation to a science instrument has not been approved.

\section{COSMIC SYSTEM}

Figure 3 is a high-level overview of the COSMIC system. The space segment consists of the eight LEO satellites and the GPS constellation. The data from the eight satellites are transmitted to the high latitude ground stations in Fairbanks and Kiruna probably via L-band. From these receive ground stations the data are forwarded to the COSMIC Data Analysis and Archive Center (CDAAC) in Boulder, Colorado. The CDAAC also receives data from a global network of ground GPS and TBB receiving sites (the so-called fiducial network). Data from the 
fiducial network will not flow directly to the CDAAC, but rather to a fiducial network operations facility which is not shown because it will not be operated directly by COSMIC. The CDAAC will process and archive all received data and forward these data to scientific and operational users. All data and products at CDAAC will also be copied to the Taiwan Analysis Center for COSMIC (TACC). TACC will also perform its own analysis of the data and distribute its products and CDAAC products to the user community in Taiwan. NSPO mission operations will be responsible for constellation operations. Satellite and payload commanding will be handled by NSPO via S-bad uplink from two Taiwanese ground stations. The satellites, the ground fiducial network, and the CDAAC will be described in more detail in the following sections.

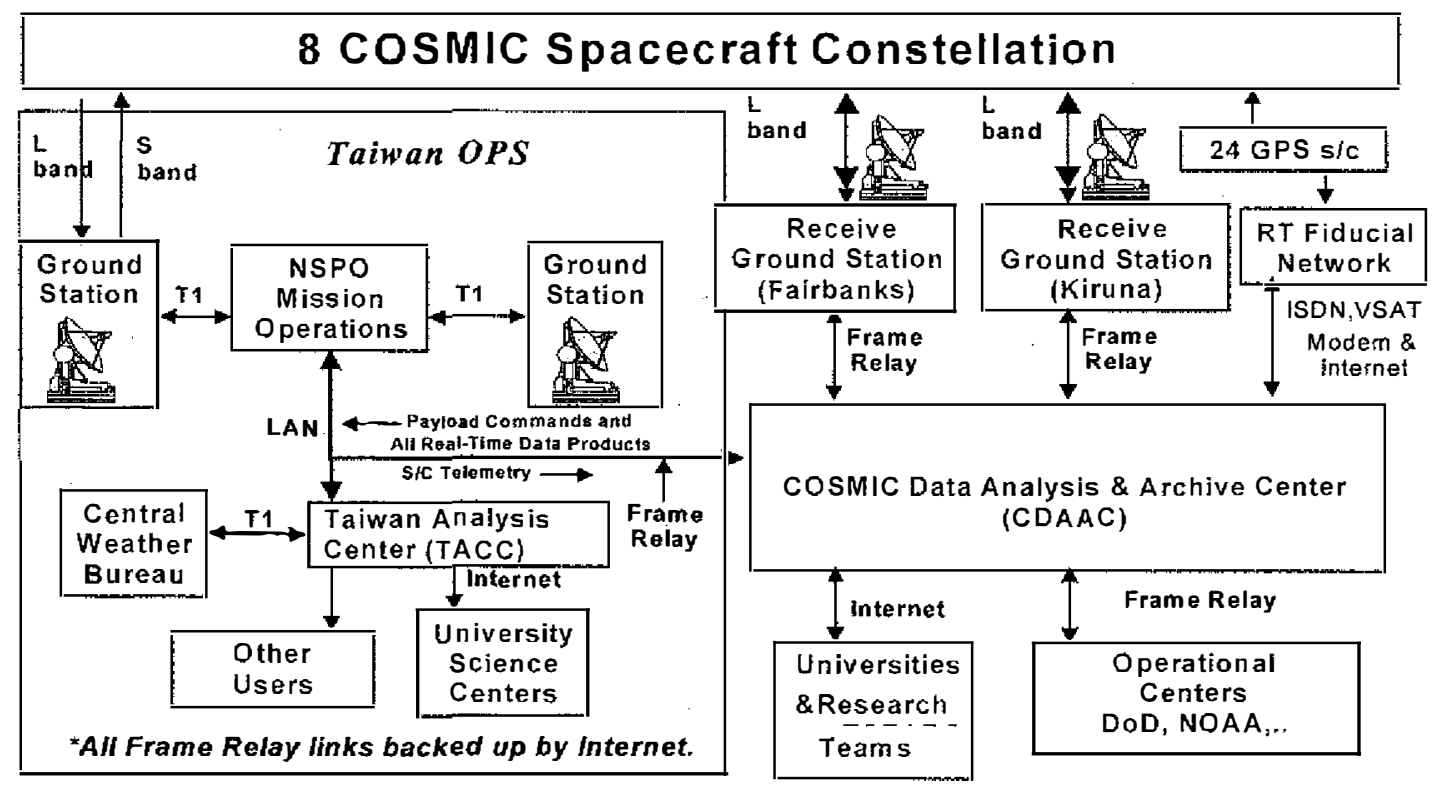

Fig. 3. COSMIC System Overview.

\subsection{The COSMIC Satellites}

During the initial COSMIC study phase a preliminary design of the COSMIC spacecraft was completed. The satellite manufacturer has yet to be selected and the preliminary design will likely be changed considerably. The total spacecraft mass is expected to be $\sim 40 \mathrm{~kg}$ (including propellant). The spacecraft shall be 3 -axis stabilized and shall feature a monopropellant (hydrazine) propulsion system, for orbit insertion, maintenance and de-orbiting. The overall propellant mass is expected to be about $8 \mathrm{~kg}$, sufficient for a total velocity change of $450 \mathrm{~m} / \mathrm{s}$. Solar panels shall be sized to provide $55 \mathrm{~W}$ of orbit average power to the spacecraft. The COSMIC spacecraft shall be designed to launch eight spacecraft on a single launch vehicle.

The Attitude Control System (ACS) is planned to be a pitch-momentum biased system. The vehicle's attitude is primarily nadir with fixed forward and aft facing GPS antennas. There 
are three orthogonal torque rods to control the roll and yaw axis, as well as control and dissipate momentum built up in the pitch oriented reaction wheel. There is one earth sensor to achieve $\sim 0.1$ degree pitch knowledge required for the TIP, and a magnetometer to achieve three axis determination for the mission to $\sim 1$ degree. The spacecraft also includes a solid state Inertial Reference Unit (IRU) to null out rates during propulsion orbit raise and orbit maintenance maneuvers.

The power subsystem is based on two Gallium-Arsenide based solar arrays in a fixed deployed configuration. The solar array will not be articulated for cost, complexity, and aerodynamic drag considerations. The batteries are Li-Ion based 14.4 volt, $15 \mathrm{Amp}-\mathrm{Hr}$, aerospace cells. Power distribution and battery charging are accomplished with solid state, analog control looped based, switches (HexFETs), and monitoring systems to ensure proper power utilization and safe hold modes. The power distribution system also contains the propulsion valve drivers in order to operate the propulsion nozzles in a pulse width modulated method.

Propulsion is a blowdown hydrazine system with a cluster of four axial canted thrusters. These thrusters are operated in a pulse-width-modulated method in order to achieve three-axis control, and the required velocity changes (delta- $\mathrm{V}$ ) for the orbit raise and maintenance maneuvers. Fuel mass fraction for this mission is approximately $20 \%$ or $8 \mathrm{~kg}$ of hydrazine.

The flight computer and solid state recorder shall be based on commercially produced, radiation tested or radiation-hardened low power parts. The flight computer contains a PowerPC microprocessor, EEPROM, PCI communications bus and system memory. The solid state recorder, on the PCI bus, contains approximately 64Mbytes of store and forward memory for the instruments. The communications interface card, also on the PCI bus, contains all the communications protocols and command decoding to orchestrate the physical layer communications and low level relay commands.

The current design includes a TT\&C system that consists of an S-Band FSK receiver designed for data rates of $32 \mathrm{kbps}$. The telemetry transmitter is a $2 \mathrm{Mbps} \mathrm{L}-\mathrm{Band}$ BPSK. The LBand ( $1690 \mathrm{MHz}$ to $1710 \mathrm{MHz}$ ) is recommended for use because this part of the spectrum is already allocated for spacecraft meteorology data downlink.

The software will incorporate a real-time operating system. Most software development will be required for the attitude control modes (safe hold, propulsion maneuvers, nadir following, etc.), while a smaller portion is for the state-of-health, command, payload operations, data communications, and compact file system orchestration. Additional capability must be developed for software uplink and reprogramming.

\subsection{The COSMIC Orbit Constellation}

Launch of eight COSMIC micro-satellites into LEO is planned for 2003. The desired orbits are 72 degrees inclination and $800 \mathrm{~km}$ circular, with all spacecraft in their own orbital plane spaced approximately 23 degrees apart in ascending node. Alternative orbits are also feasible and constellation design is still in progress. The satellites will be pointed at nadir to ensure that the high-gain occultation antennas are directed to within $+/-5$ degrees of the Earth's limb. The satellites will maintain a fixed yaw orientation with the high-gain antennas pointing in the velocity and anti-velocity directions. Attitude will be known to $+/-1$ degree at all times. 


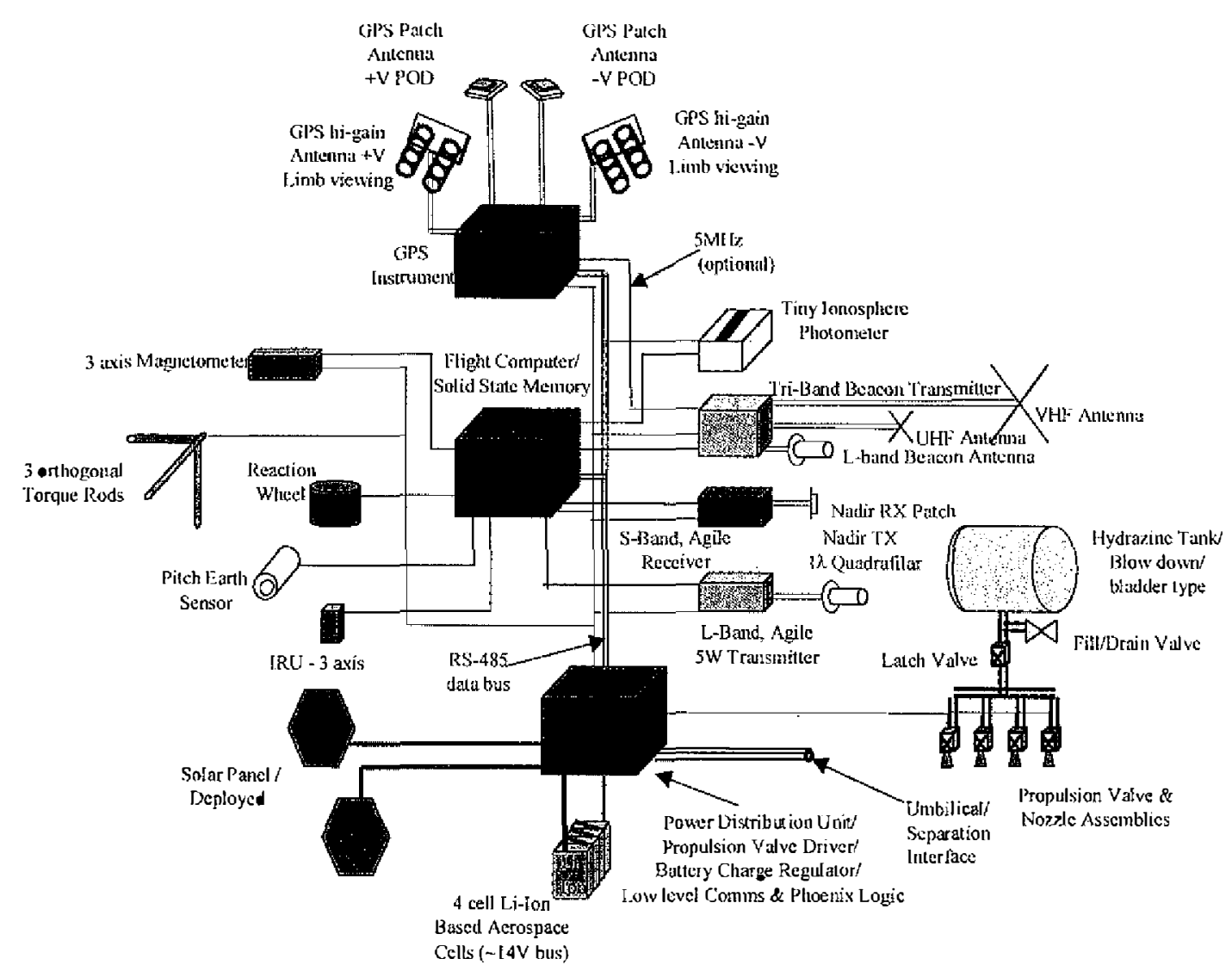

Fig. 4. Preliminary design diagram of the main COSMIC satellite components.

Depending on the final constellation a global snapshot can be achieved every 100 minutes. The system will retrieve $-4,000$ vertical profiles of the atmosphere each day. The result will be meteorological data at heights from approximately $60 \mathrm{~km}$ to the surface and ionospheric data from approximately 90 to $800 \mathrm{~km}$.

The COSMIC orbits will be chosen to balance mission science requirements with other mission design and cost priorities. The main scientific objective for the COSMIC mission is to provide the most useful atmospheric and ionospheric data products possible to the science community in near real time (within 2-3 hours of the observations). The primary science requirements recommended at the COSMIC science workshop in Taiwan (February, 1998) state a desire to maximize the number and the global refresh rate of the radio occultations produced by the constellation. Maximizing the number of occultations equates to increasing the number of satellites in orbit. One LEO satellite can acquire approximately 500 rising and setting occultations from the GPS constellation in one day. Maximizing the global refresh rate of the occultations requires spreading out the satellite orbits equally in ascending node. This allows adequate sampling of the diurnal signal of Earth's atmosphere and ionosphere every orbit. Additional science requirements that helped establish guidelines for the COSMIC constellation 
include: orbit inclinations large enough to give adequate polar coverage, and orbit altitudes that are above the majority of the ionosphere even at solar maximum to improve ionospheric occultations and minimize drag.

The constellation design and analysis for this mission involves complex trade-offs between the science requirements and many other issues. These issues are related to program cost, ground station download coverage and data latency, mass to orbit launch capability, satellite deployment strategy, satellite fuel budget, and the design of the propulsion and attitude control systems. Because cost is the major constraint, the design emphasized the selection of a constellation that could simultaneously meet the science objectives and that could also be deployed with a single launch vehicle. The least expensive way to phase multiple satellites in ascending node when using a single launcher is to precess the orbits differentially over a period of time. Differential precession between orbits with different altitudes or inclinations occurs due to Earth oblateness ("J2") effects (i.e., Space Handbook, 1985). This requires a well thought out constellation deployment strategy and additional fuel to change the altitudes and/or inclinations of the orbits. After numerous trade studies, an initial design for the COSMIC constellation was completed and is summarized in Table 1.

\subsection{COSMIC Payloads}

The primary COSMIC instrument will be an advanced GPS receiver developed by JPL. This receiver is a heritage design of an earlier JPL-developed instrument that was flown aboard the MicroLab-1 satellite during GPS/MET. The COSMIC GPS receivers will be able to track all GPS satellites in view simultaneously with two or more occulting satellites. It will operate fully autonomously, scheduling when to track which satellites and at what sampling rate based on its own known position and those of the GPS satellites. The instrument will report high-rate $(50$, or even $100 \mathrm{~Hz}$ ) dual frequency carrier phase change on the occulting links with sub-mm precision for accurate high resolution profiling. Lower rate $(0.1 \mathrm{~Hz})$ phase measurements of all satellites in view will be collected for $5-10 \mathrm{~cm}$-level orbit determination. The receiver has to measure both GPS carrier frequencies to separate the frequency dependent (dispersive) ionospheric delay from the non-dispersive refractive delay of the neutral atmosphere. In addi-

Table 2. Summary of desired COSMIC satellite constellation parameters.

\begin{tabular}{|l|l|}
\hline Design life & 5 years \\
\hline Number of spacecraft & 8 \\
\hline Number of planes & 8 \\
\hline Spacecraft mass & 40 kilograms \\
\hline Semi-major axis of parking orbit & 400 kilometers \\
\hline Semi-major axis of operational orbit & 800 kilometers \\
\hline Orbit Eccentricity & 0, circular \\
\hline Orbit Inclination & 72 degrees \\
\hline Plane separation in Ascending Node & 22.5 degrees \\
\hline Spacecraft separation in Mean Anomaly & 45.0 degrees \\
\hline Differential precession drift time & $\sim 300$ days \\
\hline Delta-V fuel load (including reserve) & $\sim 455$ meters/second \\
\hline
\end{tabular}


tion to accurate phase measurements, the GPS instrument can also record GPS signal amplitudes for on-orbit ionospheric scintillation monitoring and correction of signal diffraction effects in post-processing.

Two additional instruments will orbit on COSMIC: the tiny ionospheric photometer (TIP) and a tri-band beacon (TBB) transmitter, both built at the Naval Research Laboratory (NRL). The TIP is a nadir viewing $135.6 \mathrm{~nm}$ photometer, which measures naturally occurring extreme ultraviolet airglow. From TIP measurements the nighttime electron density at the peak of the F2 layer can be computed with high spatial resolution along the satellite tracks.

The TBB transmits three frequencies (150, 400 and $1067 \mathrm{MHz}$ ), which can be tracked by receivers on the ground or aboard other satellites. Measured phase differences between the different frequencies yield total electron content along the transmitter to receiver paths. A COSMIC transmitter pass over a chain of beacon receivers on the ground will provide data for high-resolution two-dimensional tomographic measurements of electron density. Amplitude fluctuations of the TBB data will be used for scintillation monitoring. The $1067 \mathrm{MHz} \mathrm{TBB}$ carrier frequency will be modulated to carry a low-rate data stream of on-orbit determined GPS scintillation parameters and important spacecraft health information.

TIP and TBB will complement the primary GPS occultation observations to provide much more complete three-dimensional fields of electron density in the ionosphere. The observational requirements for data to be received at CDAAC during the COSMIC mission are summarized in Table 3. These requirements are driven by the science requirements from Table 1.

\subsection{Ground Segments of COSMIC}

The COSMIC system requires significant ground infrastructure. The key components of the COSMIC system are shown in Fig. 3. The main communication links are shown in Fig. 5.

Each COSMIC satellite dumps its data to one of two high-latitude Earth stations once per orbit every 100 minutes for immediate transmission to the near real-time CDAAC at UCAR.

Table 3. COSMIC Observational Requirements.

\begin{tabular}{|l|l|}
\hline \multicolumn{1}{|c|}{ Measurement Parameter } & \multicolumn{1}{c|}{ Requirement } \\
\hline Number of occultations & $\sim 4000$ soundingsiday \\
\hline Distribution & Global \\
\hline L1 / L2 phase measurement & $\sim 2 \mathrm{~mm}(10$-sec, iono-free data for POD) \\
\hline GPS phase sampling rate & $\begin{array}{l}0.1-50 \mathrm{~Hz} \text { (the possible need for } 100 \mathrm{~Hz} \\
\text { sampling in the lower troposphere is still } \\
\text { under investigation) }\end{array}$ \\
\hline GPS vertical range neutral atmosphere & surface $-60 \mathrm{~km}(50 \mathrm{~Hz}$ sampling) \\
\hline GPS vertical range ionosphere & $90-800 \mathrm{~km}(10 \mathrm{~Hz}$ sampling) \\
\hline TBB phase measurement & $<32 \mathrm{~mm}$ ( $150 \mathrm{MHz}$ (ground receiver) \\
\hline TBB sampling rate & $>50 \mathrm{~Hz}$ (ground receiver) \\
\hline TIP measurement & $<10 \%$ (uncertainty in photon count) \\
\hline TIP footprint & $125 \times 25 \mathrm{~km}$ (at $400 \mathrm{~km}$ height of $\mathrm{F} 2$ layer) \\
\hline TIP resolution & $0.1-10 \mathrm{sec}$ averaging \\
\hline Magnetometer & $10 \mathrm{nT}$ precision, $500 \mathrm{nT}$ accuracy \\
& (may not be included - not yet decided) \\
\hline
\end{tabular}


The CDAAC also requires data, from a global network of $\sim 25$ ground based receiver sites in near real time (within $\sim 10 \mathrm{~min}$.). These data include GPS observations, beacon transmissions from the COSMIC satellites received at the fiducial sites, and telemetry data sent via the beacon modulation to ground-based beacon receivers. The GPS data are needed to compute precise COSMIC satellite orbits, and to eliminate errors due to GPS transmitter and receiver clock oscillator instabilities. The beacon data are used for ionospheric tomography and scintillation monitoring in the vicinity of the beacon receiver site. The beacon telemetry data are used primarily for scintillation monitoring based on the GPS data received on orbit.

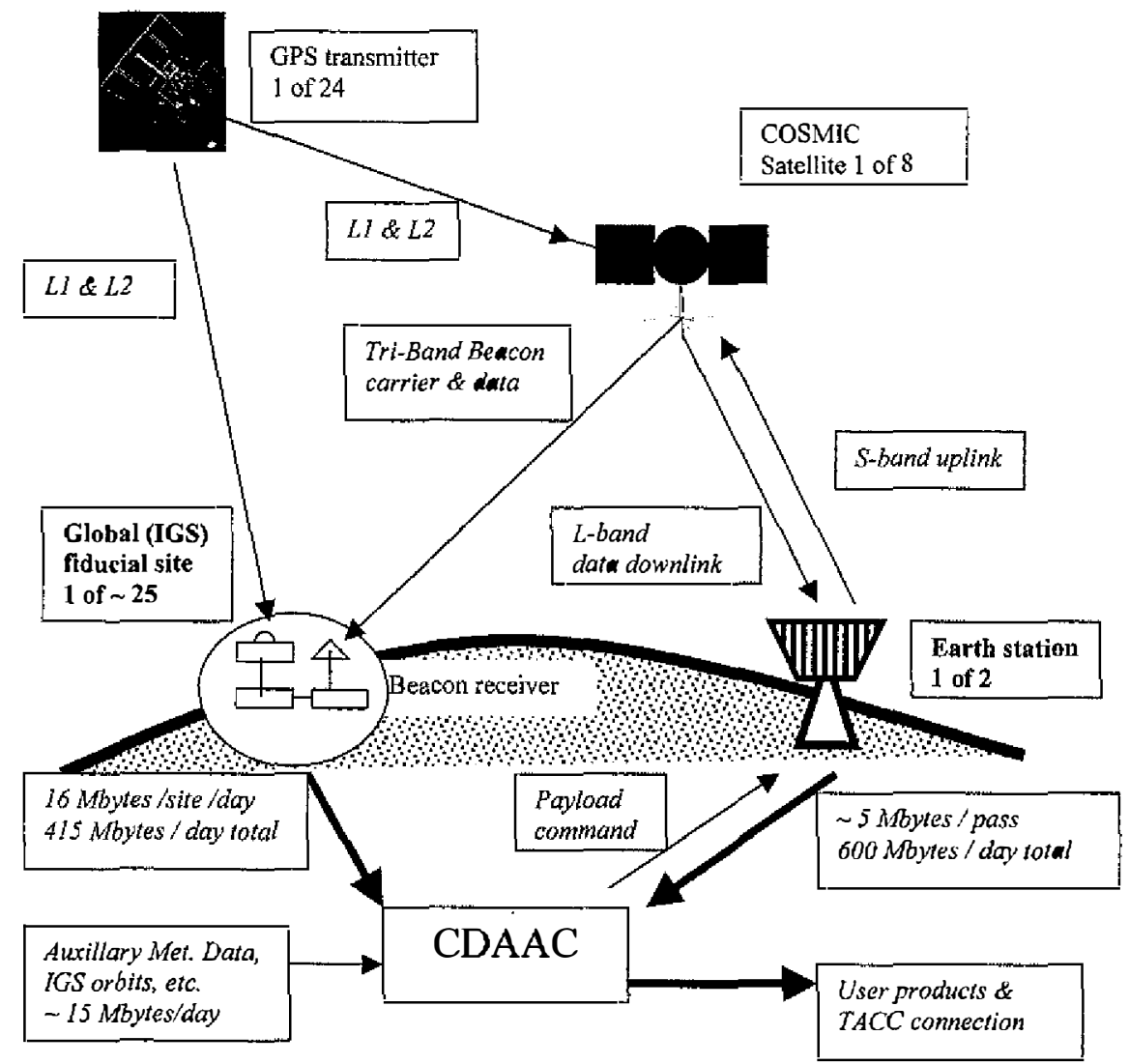

Fig. 5. Major COSMIC components, communications links data types and volumes. Data volume for the fiducial sites was computed based on the assumption of 1-sec dual frequency phase, pseudorange and amplitude GPS data, collected from an average of 8 satellites. We assumed compressed RINEX files and about 4 Mbytes of TBB data per fiducial site. For the LEO data we assumed $400050 \mathrm{~Hz}$ soundings/day with a data volume of 100 kbytes for one ionospheric and atmospheric sounding. This adds up to $400 \mathrm{Mbytes} /$ day to which we added a $50 \%$ contingency because of uncertainties in the occultation data format and sampling rate. 
The CDAAC analyzes all data and monitors payload performance. Data and higher level products will be provided to researchers and operational centers worldwide. (See Table 4 for our definition of the different product levels.) All data and products will be copied to the TACC in Taiwan. NSPO will be responsible for mission operation and control including all satellite uploads from the two Taiwanese ground stations, and for the distribution of data and products to the Taiwanese operational and science communities. Next we describe the COSMIC fiducial network design, the Earth stations, and the CDAAC.

\subsubsection{The Fiducial Network}

To compute accurate radio occultation inversions it is necessary to remove the drifts of the GPS transmitter and receiver clocks from the raw phase data. This can be done with common mode double difference viewing of the LEO and ground GPS data as illustrated in Fig. 6.

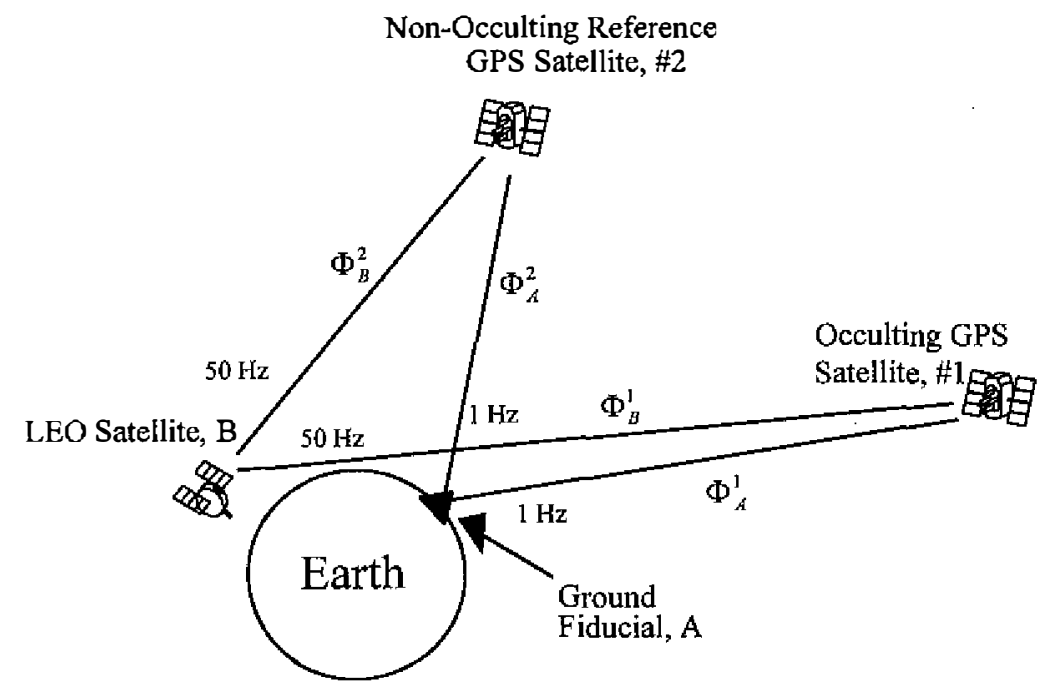

Fig. 6. Shows the use of a fiducial site for forming a double difference. The fiducial sites will $\log$ GPS data $\left(\Phi_{\mathrm{A}}^{2}\right.$ and $\left.\Phi_{\mathrm{A}}^{1}\right)$ at 1 second while the LEO will track the occulting and reference satellites at $50 \mathrm{~Hz}$ or maybe even at a higher rate $\left(\Phi_{\mathrm{B}}^{2}\right.$ and $\left.\Phi_{\mathrm{B}}^{1}\right)$.

COSMIC and several other upcoming satellite missions (including Orsted, SUNSAT, SRTM, CHAMP, SAC-C, GRACE) plan to carry GPS receivers that will acquire radio occultation measurements of the Earth's atmosphere and ionosphere. During the GPS/MET mission a network of 6 sites (Potsdam, Germany: POTS, Fairbanks, Alaska: FAIR, Goldstone, California: GOLD, Kokee Park, Hawaii: KOKB, Tidbindilla, Australia: TID2, and McMurdo, Antartica : MCM4) was operated by JPL. This network must be expanded and improved to capture all future radio occultation data.

Simulation software was used to compute all double difference combinations between the GPS satellites as viewed from the LEO satellites and ground sites. We found that all occulta- 
tions can be double differenced with a 13 station fiducial network when a 15-degree elevation cutoff mask is used. At least 20 stations are required to capture all occultations with a 30 degree elevation mask.

It was also found that a LEO network of 8 evenly distributed satellites can provide complete double difference coverage if the LEOs can track all GPS satellites in view that are above the $F$ layer of the ionosphere $(\sim 400 \mathrm{~km})$. This approach would permit use of the double difference technique without the requirement for a $1-\mathrm{Hz}$ ground fiducial network (simply replace ground fiducial site A in Fig. 6 with another COSMIC LEO satellite). For COSMIC this approach is considered experimental because it will increase the time needed to analyze the occultations. With the ground fiducial approach only data from one LEO and the ground network are required for profile analysis. If the ground fiducial data are available with no more than 10 minutes latency (as is planned for COSMIC) then the occultation data from each data dump at the Earth stations can be differenced with fiducial data within minutes of reception at the CDAAC. For processing with orbiting reference data, on the other hand one has to wait for one entire orbital period after each download (100 minutes) before the data can be differenced to eliminate clock errors.

\subsubsection{Impact of high frequency ground fiducial multipath}

A potential problem with using ground based GPS data to remove unwanted clock signal from occultation data is the presence of high frequency multipath. Evidence of site multipath can be seen in post-fit residual from GPS/MET fiducial phase data. Figure 7 shows evidence of site multipath at MCM4 and TID2 from the analysis of GPS/MET $1 \mathrm{~Hz}$ LC (linear combination - ionosphere free) phase data on consecutive days in 1997: 97.034 and 97.035. Multipath is expected to repeat itself from one sidereal day to the next. The correlation between the residuals on the consecutive days is apparent evidence of site multipath. Figure 7 shows temperature retrievals (using ground data from TID2, MCM4, and KOKB) for a GPS/MET occultation that occurred during the same time period (on 97.035) as shown in Fig. 7. The curve for MCM4 deviates from the other curves by nearly 4 degrees $C$ at $30 \mathrm{~km}$. This is caused by the multipath of PRN 10 (elevation of 7 degrees), which has a velocity magnitude of nearly $1 \mathrm{~cm} /$ sec. The curves for TID2 and KOKB differ by $\sim 0.5$ degrees $C$ at $30 \mathrm{~km}$. This difference is caused by a more typical level of multipath (from PRN 05, elevation of 30 degrees) with a magnitude of near $1 \mathrm{~mm} / \mathrm{sec}$. The impact of high frequency multipath on occultation retrievals is significant and the subject of further study.

\subsubsection{High accuracy clocks for the COSMIC fiducial network}

This section discusses the required clock quality for receivers that are used for GPS radio occultation processing. This discussion concentrates on un-modeled errors in the rate-of-change of the excess phase (velocity), which can have a large effect on occultation retrieval accuracy. The magnitude and period of the velocity error are important. Longer period velocity errors (nearly constant over the occultation) will cause larger retrieval errors than shorter period velocity errors of similar magnitude because the retrieval is an integral process.

The tracking configuration is assumed (Fig. 6) to consist of a low Earth orbiting (LEO) 

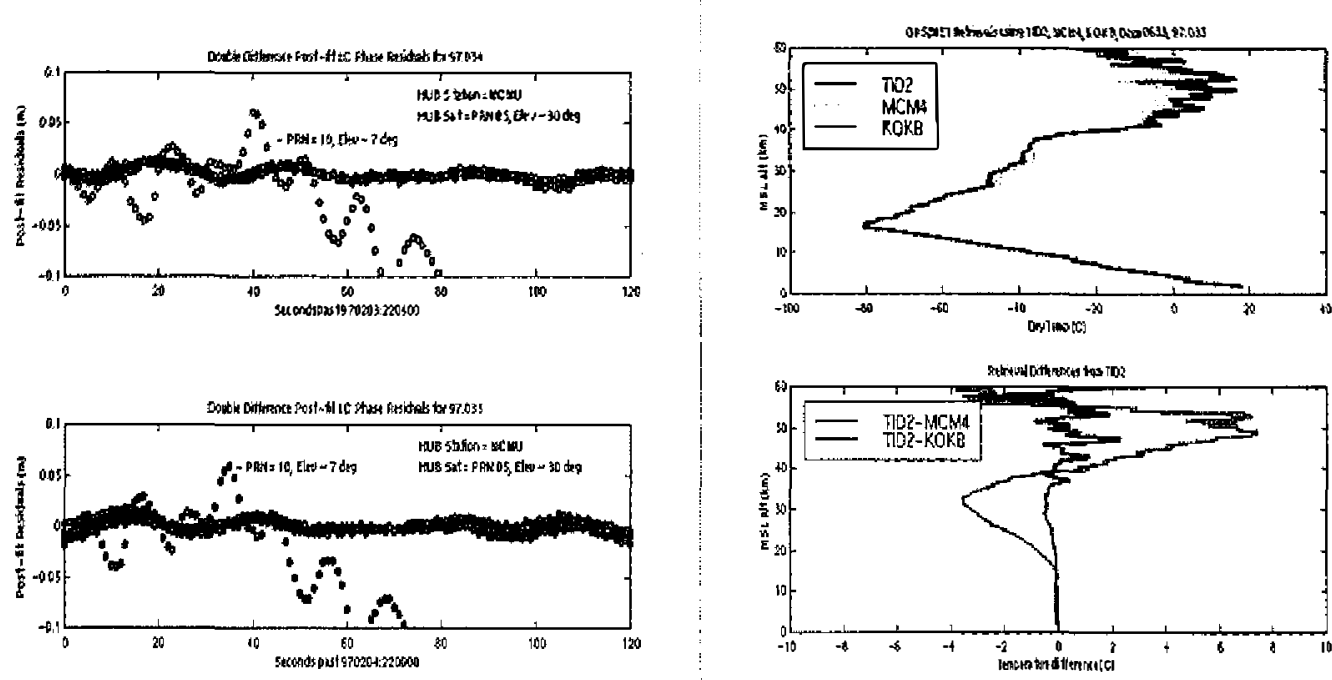

Fig. 7. The left two panels show evidence of site multipath from the analysis of GPS/MET I Hz LC phase data on consecutive days. The top panel shows double difference phase residuals between MCM4 and TIDB over a 2 minute interval on 97.034 (YY.DOY, year and day of year). The bottom panel shows the residuals for the same sites on the following day (97.035), but shifted 4 minutes earlier. The high correlation of the residuals from 1 sidereal day to the next is evidence of multipath. The right top panel shows a plot of GPS/MET temperature inversions using 3 different fiducial sites: TID2, MCM4, and KOKB. The right bottom panel shows tem perature differences.

receiver tracking an occulting satellite and a reference satellite at high rate $(50 \mathrm{~Hz})$ and a ground receiver tracking the same satellites at a lower rate $(1 \mathrm{~Hz})$. Selective Availability (SA) error has an impact on how good the clocks need to be. The current plan for COSMIC occultation processing is to form double differences of the LEO and ground observations to remove clock errors. Most GPS processing software forms differences of the observations with common (simultaneous) receive times. However, SA is only completely removed if the data are differenced using common transmit times. The residual SA velocity error that remains when using common receive times can be as large as $0.4 \mathrm{~mm} / \mathrm{sec}$ ( 3 sigma) and is essentially constant for the entire occultation period. To use common transmit times, one must interpolate the data in time so that the transmit times for the observations are simultaneous. This process trades the errors in the dithered satellite clocks (SA) for the errors in the receiver clocks. Using common transmit times will make the receive times (at one receiver) for the two satellites nonsimultaneous by as much as 0.02 seconds. Therefore, if an average frequency drift is present in the receiver clock during the occultation, then a residual velocity error will remain after the 
two non- simultaneous observations are differenced. This residual velocity error will be proportional to the product of the frequency drift and receive time off set $(0.02 \mathrm{sec})$. To ensure that the residual velocity error during a 60 second occultation period is less than $0.05 \mathrm{~mm} / \mathrm{sec}$, the average frequency drift during the occultation should be less than $0.0125 \mathrm{~Hz} / \mathrm{sec}$.

Since ground tracking is planned at $1 \mathrm{~Hz}$, the $\Delta f / f$ frequency stability of the oscillator (over 1 second) also generates some residual velocity error because the ground data must be interpolated (to $50 \mathrm{~Hz}$ ) to different receive times. For an oscillator with a stability of 1 part in $10^{10}$ (over 1 second averaging time), this residual velocity error can have a magnitude of nearly $1 \mathrm{~mm} / \mathrm{sec}$, but it will also have a period of about 1 second. Because of the short period of this velocity error, it is expected to generate a small bias error in the occultation retrieval. To estimate the magnitude of this short period velocity error, a simulation was performed by adding a $1 \mathrm{~mm} / \mathrm{sec}$ sinusoid (with 1 second period) to GPS/MET excess phase data, computing the retrieval, and comparing the modified and original retrievals. The simulation showed that this $1 \mathrm{~mm} / \mathrm{sec}$ error generated oscillatory errors of 1 degree $C$ magnitude at $\sim 35 \mathrm{~km}$ and up to 5 degrees $\mathrm{C}$ above $50 \mathrm{~km}$. This residual velocity error is essentially eliminated (reduced to near $0.05 \mathrm{~mm} / \mathrm{sec}$ ) if the stability of the ground oscillator is 5 parts in $10^{11}$ (over 1 second averaging time). Because stable oscillators are expensive, the effect of this short period velocity error should be studied further and compared against the effects of other error sources.

Good clocks are only required for the ground receivers and not the LEO clock. This is because the observations can actually be formed (differenced) using simultaneous receive times at the LEO (which means the LEO clock error is removed completely by differencing) and non-simultaneous receive times on the ground. Since the LEO can use common receive times, a GPS-steered clock will be adequate for occultation processing.

In summary we conclude that high-quality oscillators shall be installed at the fiducial sites. Oscillators should as good as 5 parts in $10^{\prime \prime}$ over 1 second averaging time, with frequency drifts of less than $0.0125 \mathrm{~Hz} / \mathrm{sec}$ during the duration of a neutral atmosphere occultation. Alternatively, instead of double differencing, one can estimate SA clock dither at every second with data from the ground based tracking network. However to avoid delays in the analysis of the COSMIC data through this additional processing step we are currently planning to use the double differencing technique for clock error removal.

\subsubsection{The beacon receiver ground network}

Each COSMIC satellite will transmit TBB signals. These transmissions can be received on the ground or on orbit by other spacecraft. While COSMIC presently is not yet funded to establish the ground tracking network(s) for the TBB signals we describe here the applications of a ground based beacon receiver network.

Beacon receivers shall be collocated with GPS fiducial sites wherever possible. Additional beacon receivers will be deployed for science projects along beacon receiver chains. These chains 1000-2000 km in length and aligned with the COSMIC orbit ground tracks, will include typically 5 or more receivers, spaced by several hundred $\mathrm{km}$. The beacon receivers at the COSMIC fiducial sites will have two primary functions: (1) Collect phase and amplitude TBB data from COSMIC satellites that pass overhead, and (2) receive the telemetry stream 
transmitted by the COSMIC satellites. This scintillation telemetry data along with scintillation parameters computed from the ground received beacon signals with and the GPS fiducial data shall be transmitted to CDAAC. The scintillation parameters of this data set can provide close to real time global scintillation monitoring.

Unlike the GPS ground receiver network, the number of TBB ground receivers directly impacts the satellite design. The latency of scintillation data modulated onto the TBB carrier would benef it from more globally distributed sites, because the COSMIC satellites would see the nearest ground beacon station sooner. However, a larger number of globally distributed sites will require that the TBB transmitters be powered on a larger percentage of the time. This increased TBB duty cycle impacts the satellite power budget. Figure 8 shows a possible distribution of 25 ground TBB receiver sites and the range from which these sites can be seen from the COSMIC satellites for station elevation cutoffs of 8 and 30 degrees.

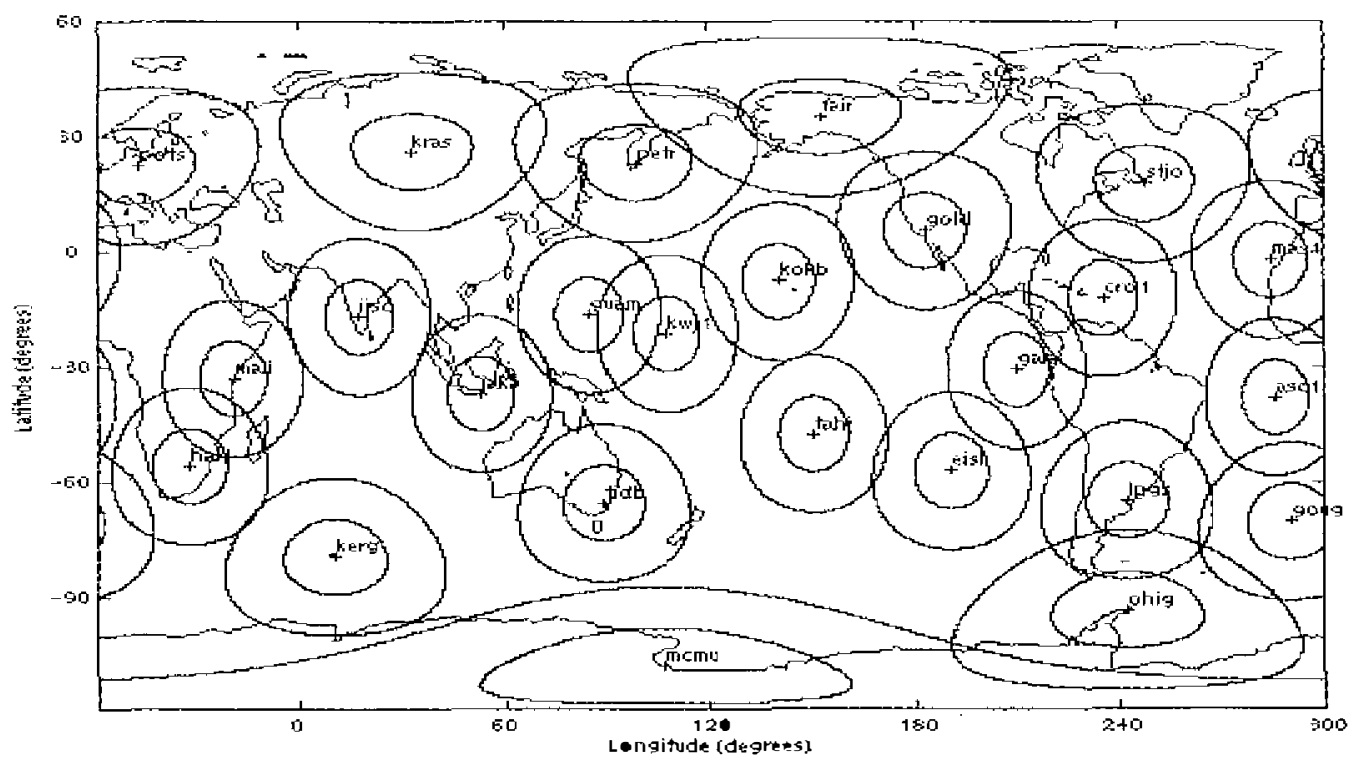

Fig. 8. Possible network 25 GPS/TBB ground sites for the COSMIC mission. The visibility masks to the COSMIC satellites are shown for elevation cutoff angles of 30 and 8 degrees.

Each time a COSMIC satellite enters within the range of one of these ground sites it will be able to transmit its modulated TBB data stream. Figure 9 shows a comparison of gaps in TBB coverage between a 25 and a 13-station globally distributed network when an 8 degree elevation angle cut-off is assumed. A gap in TBB coverage is defined as the period of time when a satellite is not in view of at least one ground station. The mean, standard deviation, and maximum values of the revisit times for each network in Fig. 9 provide a measure of the latency of the LEO scintillation data. The TBB duty cycles for the 25 station and 13 station networks are $72 \%$ and $43 \%$, respectively. A trade-off study between the above and other is- 

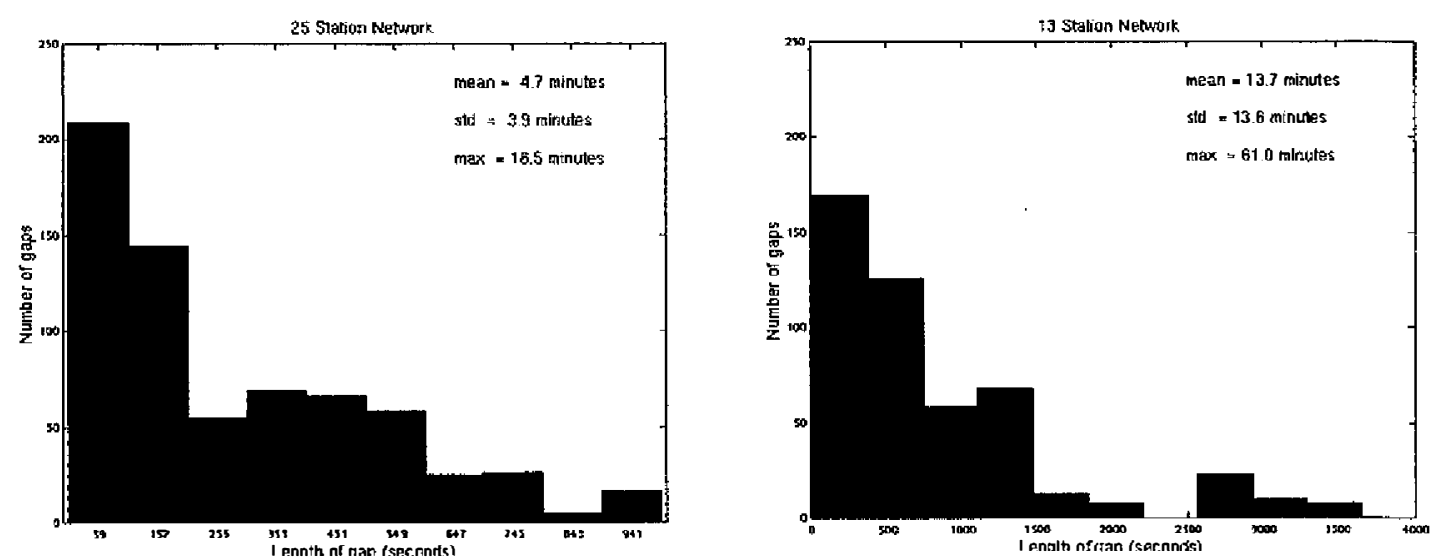

Fig. 9. This figure compares the number and duration of gaps in TBB coverage for a possible 25 station network and a smaller network of 13 stations for the duration of one day. A gap in TBB coverage is defined as the time period when the satellite is not in view of at least one ground station. An elevation cut-off angle of 8 degrees is assumed.

sues will have to be performed in the near future to determine the optimal number and distribution of GPS/TBB sites for COSMIC.

Another interesting potential use of the TBB data is water vapor measurement. The feasibility of this application still needs to be investigated. The TBB transmitter and ground receiver shall both be slaved to oscillators that are synchronized with GPS. With oscillators that are controlled in such a way and known high quality geodetic positions of the transmitter and ground receiver, measurements of the integrated water vapor delay along the line of sight of the TBB signal, may be possible. These measurements would be comparable to GPS slant water vapor measurements (Ware et al., 1997).

\subsubsection{Fiducial data communications}

To provide COSMIC products within less than 3 hours of data collection it is important to transmit the data from the fiducial network as fast as possible to the CDAAC. Several communication modes are under investigation. For locations where the Internet is not available, Very Small Aperture Terminal (VSAT) technology may be used to transfer data rapidly to the COSMIC analysis center. Several LEO missions that will be launched before have similar communications requirements as COSMIC and we are planning to collaborate with these projects on establishing the needed communications infrastructure.

In summary, the COSMIC system shall include a ground network of combined GPS and beacon receiver ground sites. About 25 GPS fiducial sites shall be operating to provide highquality dual-frequency phase and pseudorange data to the CDAAC. These data shall be available at the CDAAC within 10 minutes or less of data collection. The number of TBB receiver sites and their distribution is yet to be determined. 


\subsection{COSMIC Earth Stations}

The COSMIC remote ground stations will be located at current teleport sites in Fairbanks Alaska and Kiruna Sweden. The preliminary design of the ground stations expects L-band downlink, which may still be changed. The ground stations are program tracked to first locate the spacecraft, and then will autotrack the LEOs for best signal reception. The parabolic dish diameter is approximately 3.4 meters. These antennas may have a radome installed based on availability requirements and weather severity analysis at the proposed sites. All interfaces are commercial standards, and the RF links are designed to minimize the telecommunications costs, both on the spacecraft, as well as on the ground (Fig. 10).

Downlink characteristics for the COSMIC ground station are:

$\begin{array}{ll}\text { Antenna gain: } & 33 \mathrm{~dB} \\ \text { Receiver Frequency: } & 1680 \text { to } 1720 \mathrm{MHz} \\ \text { Polarization: } & \text { Right Hand Circular } \\ \text { System Noise Temperature: } & 175 \mathrm{C} \\ \text { Demodulator: } & \text { BPSK or QPSK on the carrier } \\ \text { Data rates supported: } & \text { Up to 2 Mbps } \\ \text { Packet Structure: } & \text { CCSDS compatible } \\ \text { Data decoding: } & \text { Reed Solomon Decoding }\end{array}$

Uplink characteristics

Antenna gain

$34.5 \mathrm{~dB}$

Transmitter Frequency

2025 to $2120 \mathrm{MHz}$

High Power Amplifier:

20 Watts

Polarization:

Right Hand Circular

Modulator:

Data rates supported:

FSK on carrier

Packet Structure:

Up to $32 \mathrm{kbps}$

Data encoding

CCSDS compatible with B-C-H encoding

Manchester

\subsection{COSMIC Data Analysis and Archive Center}

The CDAAC will be responsible for analysis of the COSMIC data. CDAAC will compute two solutions: A near-real-time solution for weather and space weather monitoring and forecasting applications, and a more accurate and better-validated post-processed solution for climate and atmospheric research. One important goal of the CDAAC is delivery of highest quality global data products within 3 hours to the operational and science community, to demonstrate the value of this data set to weather prediction and space weather monitoring. Improved reliability of quasi-operational near-real-time data products from CDAAC will require infrastructure enhancements that are presently sought from several US agencies.

As soon as the data from a 100-minute orbit have been dumped by one of the satellites at an Earth station they are forwarded to the CDAAC and analyzed. There will be $\sim 115$ such data dumps/day, on average one every 12.5 minutes. The $\sim 5$ Mbytes of data from each dump is 


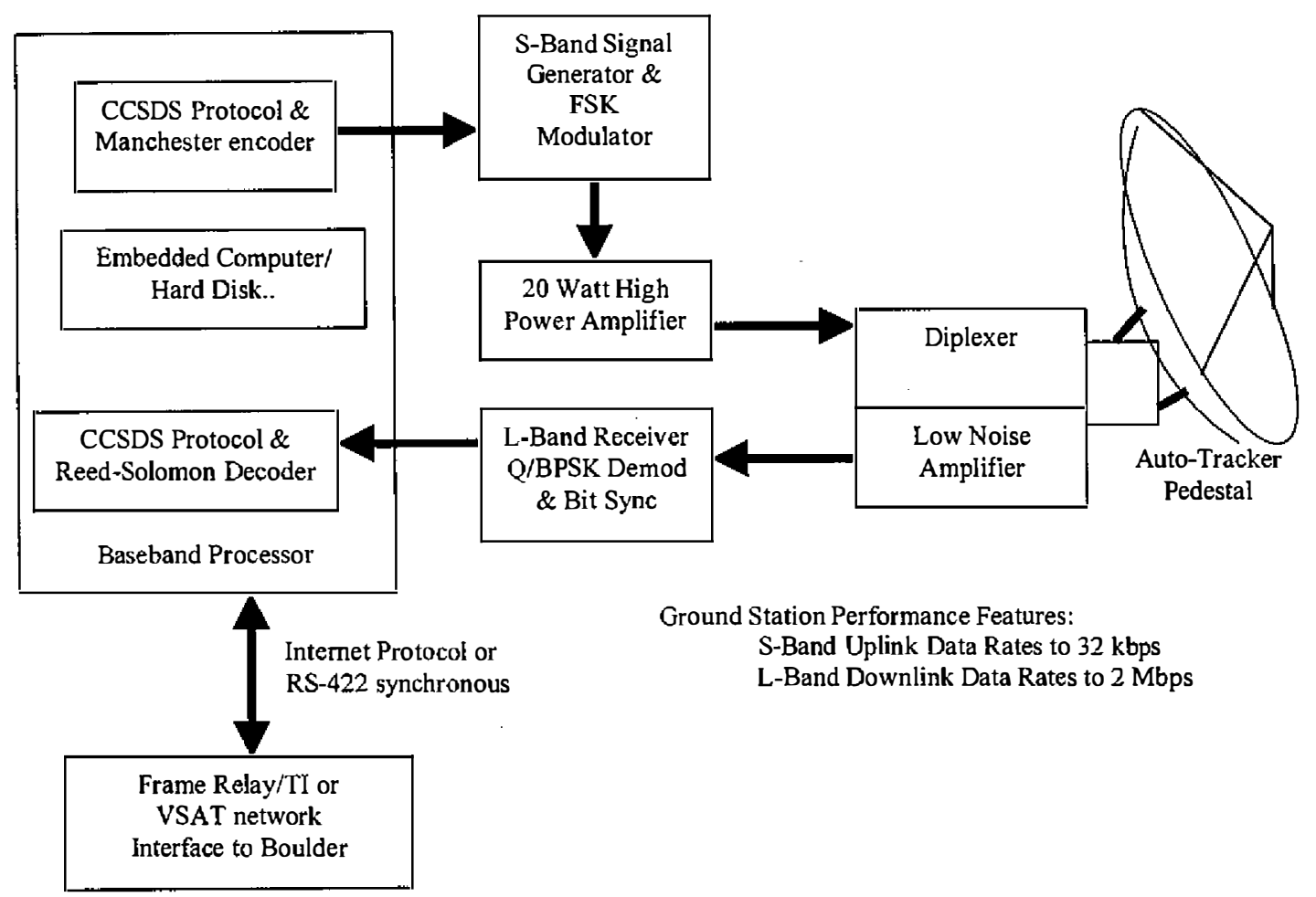

Fig. I0. Schematic of COSMIC earth station.

expected to appear at CDAAC within 10 minutes of reception at the Earth station. Since we also expect to receive the global fiducial data within 10 minutes of data collection, all the information that is required to process the occultation data is available at CDAAC with an average age of the data of $(100 / 2+10)=60$ minutes. Within $\sim 40$ minutes of data arrival, the analysis center will provide several higher level products. Profiles of temperature, pressure, humidity, refractivity, and refractive bending angles will be computed in the neutral atmosphere at an average rate of $\sim 160$ profiles/hour. In the ionosphere the CDAAC will compute profiles of electron density at a similar rate. Profiling analysis will require COSMIC satellite POD. Line of sight (biased) TEC measurements from all GPS-to-COSMIC and ground-tospace links will also be made available. Data from the TIP and TBB instruments will be processed. If they improve the results, TIP data will be included in the profiling inversion of the nighttime ionospheric occultation data. TBB will presumably be analyzed with occultation, TIP, and ground based GPS data, to obtain tomographic solutions of the global ionosphere using a Kalman filter approach (Rius et al., 1997, Howe et al., 1998). Current plans call for this tomographic analysis to be carried out not at CDAAC, but rather by other space weather analysis groups.

Products shall be transmitted via Internet to researchers, educators and operational users for assimilation into numerical models, and they will be archived for further research and education applications. The total archived data volume including raw data and higher leveI 
products is expected to be $\sim 3$ Gbytes/day. The data from the COSMIC scientific experiment and CDAAC products will be made available to all interested parties in all countries, free of charge or at the marginal cost of reproduction and distribution.

\subsubsection{CDAAC Functional Overview}

We plan to begin development of the CDAAC by mid-99 (calendar year), to be ready for the data stream from the satellites and the ground reference fiducial sites by the scheduled launch date in 2003. CDAAC will perform the following primary functions:

* Payload monitoring and control

* Incoming data quality checking

* Scientific data inversion

* Product validation

* Data distribution and archiving

Figure 11 illustrates the main functions of the CDAAC. Satellite data are received from the COSMIC polar Earth stations. This data stream consists primarily of science data but also contains spacecraft and payload health and status data. The CDAAC also receives data from the global fiducial network of GPS and TBB receiver sites. Additional meteorological data from global models are required for water vapor profiling and for validation of the results.

To carry out its payload control monitoring functions, the CDAAC must strip relevant health information from the Earth station data stream. This data will provide a first indication if any instruments aboard the satellite are malfunctioning. Additional information on the performance of spacecraft and payload will be based on results from the scientific data analysis at the CDAAC. Payload control functions will involve scheduling of the instruments, decisions on the need for new firmware uploads, etc. The Satellite Operations Control Center (SOCC) at NSPO in Taiwan will receive status reports and requests to take specific action from the payload monitoring section of the CDAAC. The satellite health data will be routed directly to the SOCC in Taiwan, which is responsible to take the appropriate action.

CDAAC expects to receive the fiducial data from JPL, UCAR/UNAVCO, and other parties primarily responsible for collection of these data from the global network. CDAAC will reformat these data and perform some basic quality checking. Additional quality information will be provided, based on the quality of the science products. If problems are encountered the fiducial network operations center(s) will be notified with requests for specific action. CDAAC will not be interfacing directly with the fiducial sites.

By far most of the computing power and development effort will have to be invested in the scientific data analysis functions of CDAAC. Here the LEO data, the data from the fiducial network, and additional meteorological data will be combined and inverted to obtain the COSMIC data products.

The CDAAC will generate results (products) on average within 3 hours of data collection. Additional high accuracy results will be computed for climate research after the highest accuracy post-processed satellite orbits are available from the International GPS Service (IGS) 


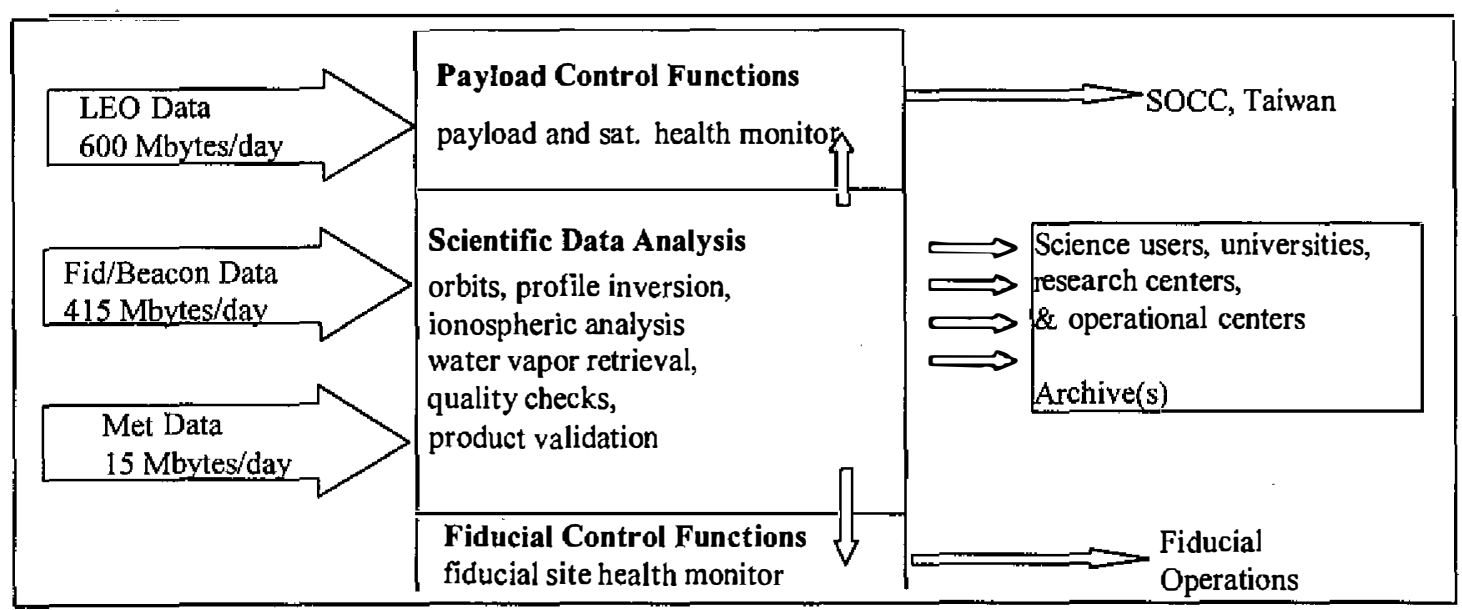

Fig. 11. Schematic of CDAAC functions.

within about 1-2 weeks after data collection. The IGS is also considering to compute LEO orbits (including COSMIC) and these orbits may be used by CDAAC for its post processing of climate data.

The current data storage plan calls for a tape-robot data archive located at UCAR. This archive will support researchers worldwide that do not require real-time data for their work. Climate data, and data for weather and space weather research, for example, will be accessed through this archive. The expected volume of raw data is about $1 \mathrm{Gbyte} /$ day. Including results and intermediate data products we expect to archive about 3 Gbytes/day. The following Table 4 summarizes the products that will be collected processed and archived. Data volumes are shown in Mbytes/day.

Table 4. CDAAC data types and estimated data rates.

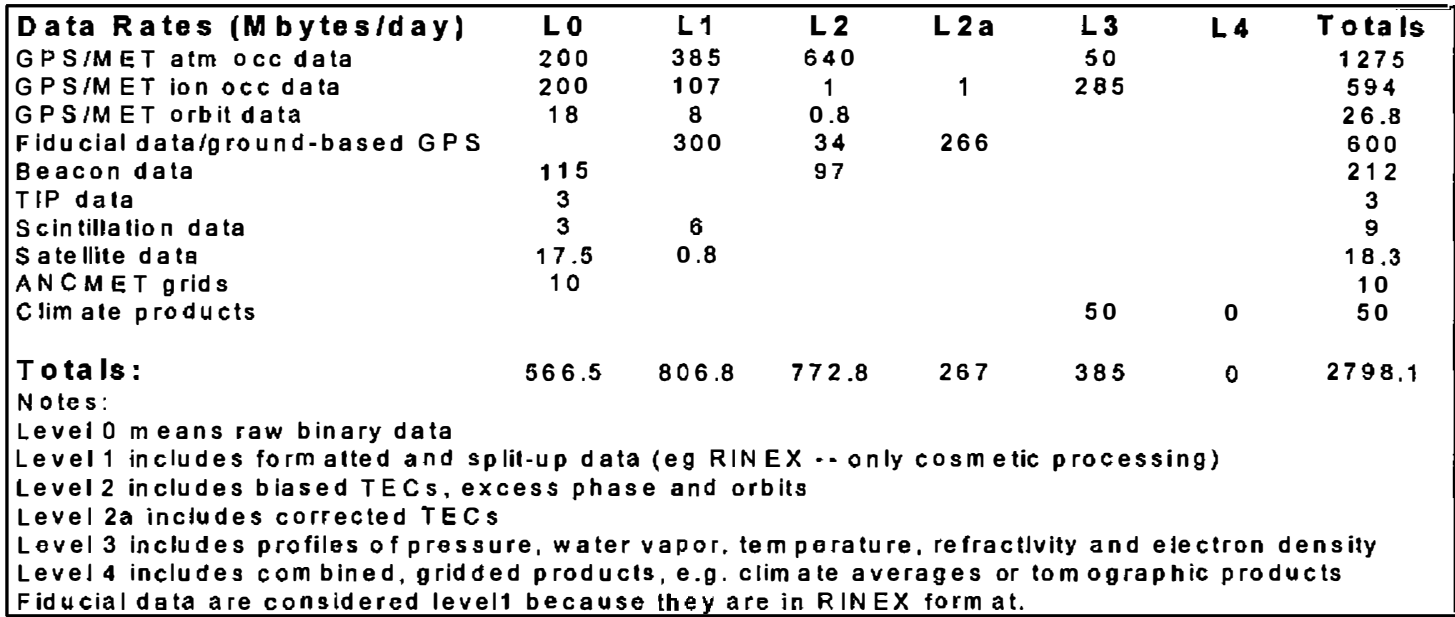




\subsubsection{CDAAC Development Phase}

Here we summarize the main development tasks that shall be accomplished for the develoment of the CDAAC.

- Design software system architecture, interfaces, and define data formats

- Develop and test orbit determination and prediction techniques for rapid results

- Improve and speed up neutral atmosphere inversion techniques and develop error models

- Develop and test new inversion techniques for the lower troposphere

- Fiducial network and multipath studies

- Test and install new ionospheric profiling techniques

- Develop techniques for analyzing scintillation data and generating global scintillation maps

- Implement and test realistic data simulators for the constellation

- Write code, document, and debug analysis system

- Test system with simulated and real data from other missions (CHAMP, SAC-C, Orsted, GRACE)

- Develop payload status analysis sof tware

- Develop payload commanding software

- Develop data validation procedures and software

- Develop database system and web interface

- Purchase, install, and network the analysis and archive hardware

- Compare test results with those from other occultation analysis groups at the Jet Propulsion Laboratory (JPL), the University of Arizona, the Max Plank Institute in Hamburg, Germany, etc.

These development tasks require significant basic research in a variety of areas including: LEO and GPS orbit prediction, lower ropospheric signal penetration, water vapor determination, diffraction and multipath correction, accounting for horizontal electron density gradients, ionospheric tomography, correction for systematic ionospheric solar cycle effects, data assimilation into numerical weather models. The COSMIC team at UCAR will investigate many of these issues. We will work closely with other groups, such as the University of Arizona, Florida State University, the University of Texas, JPL, NRL, etc. to ensure that state-ofthe-art algorithms are applied to the analysis of all COSMIC data. The most important development tasks are described in more detail in the following sections.

\subsubsection{Design System Architecture}

CDAAC system design is concerned with the data flow from the orbiting and groundbased receivers all the way to the users and archive. We will collaborate with COSMIC system engineers and payload developers on data formats, communication methods, and develop the software responsible for pulling in all the required Level 0 data at CDAAC. The software and interfaces to process all of the Level 0 data will have to be designed at a functional level. Data reception latency, required processing time, hardware requirements etc. will have to be speci- 
fied. The goal of this system architecture design is a fully automated front-to-end analysis system. CDAAC will generate products within about 40 minutes upon data reception and 3 hours of data collection on orbit. We will work with other UCAR programs on developing the system for data archiving (UNAVCO) and for data distribution to the users (Unidata). The CDAAC team will also develop a web page to display real time global snapshots of, water vapor, temperature, tropopause height, geopotential height, electron density, and scintillation. This web page will become a high-visibility and dramatic showcase for the COSMIC mission.

\subsubsection{Precision Orbits}

For precision analysis of occultation data, especially at altitudes between $30-60 \mathrm{~km}$, LEO and GPS satellite velocities have to be known to about $0.1 \mathrm{~mm} / \mathrm{s}$ or better. This requires POD techniques that have been demonstrated during the GPS/MET experiment (Schreiner et al., 1998). The challenge for CDAAC will be to achieve this orbit quality close to real time. We will investigate two approaches to obtaining high-quality orbits in real time: (1) forward propagation of orbits (orbit prediction), and (2) orbit adjustment with the most recent data from the fiducial net and from the COSMIC satellites. While (1) will provide faster results, (2) is expected to be more accurate. We need to investigate the optimal orbit strategy and implement it. CDAAC will use either the University of Berne developed Bernese software, or the commercial MicroCosmTM software for POD.

It should be noted that accurate temperature and pressure profiles between $30-60 \mathrm{~km}$, while important for climate research and other science applications, are not as important for weather prediction as data from the lower troposphere. Since temperature profiles in the lower troposphere are less sensitive to orbit errors it may be acceptable to relax real-time orbit requirements to $0.5 \mathrm{~mm} / \mathrm{s}$ while maintaining the stricter requirements for post processing of climate products.

\subsubsection{Improvements in neutral atmosphere analysis}

During GPS/MET we developed the analysis sof tware package for neutral atmospheric inversions described in Rocken et al. (1997). The CDAAC inversion sof tware will build on that experience with several key improvements. The diffraction correction code developed for GPS/MET (Gorbunov and Gurvich, 1998; Karayel and Hinson, 1997) was rather slow and seems to be sensitive to noise. This code will have to be replaced with new and improved algorithms. We also will investigate different algorithms for extracting water vapor information from refractivity. One candidate is the adoption of FSU/NCAR's 1-dimensional variational (1-DVAR) data assimilation technique for the occultation analysis system (Zou et al., 1999, Kuo et al., 1998).

GPS/MET data analysis removes the ionospheric effect for neutral atmospheric profiling by forming a model-independent linear combination of the observed $\mathrm{L} 1$ and $\mathrm{L} 2$ bending angles (Vorob'ev and Krasil'nikova 1994). This correction mode leaves a small systematic residual error, depending on the level of ionospheric activity. While this error is negligible in the lower troposphere it does affect stratospheric temperatures and it can have a long-term dependence on the 11-year solar cycle. Thus, to avoid aliasing of the solar cycle into the long-term clima- 
tological record of stratospheric temperatures from COSMIC, we will develop a model-dependent $2^{\text {nd }}$ order ionospheric correction that shall be applied in addition to the first order correction. We anticipate that this 2 nd order correction will, to some extent, be based on the global ionospheric results from COSMIC and other occultation missions.

During GPS/MET we reported that occultation refractivity was often biased significantly lower than refractivity from correlative data (the so-called $\mathrm{N}$-bias). We will pursue the investigation into this $\mathrm{N}$-bias to make sure its cause is fully understood and that it will not affect COSMIC results. This task will involve simulating realistic occultation phase and amplitude data with a phase screen model (Gorbunov and Gurvich, 1998) of the atmosphere and tracking that signal with the same firmware as used in the GPS flight receivers.

Atmospheric inhomogeneity is the dominant error source for profiles of refractivity below $20 \mathrm{~km}$, obtained with the standard Abel inversion technique. Resulting errors reach up to $10 \%$ near the surface (Kursinski et al., 1997). One way to avoid this error is to avoid the Abel inversion, and to assimilate profiles of bending angles into numerical weather models (Eyre, 1994, Zou et al., 1999, 2000). The CDAAC atmospheric scientist will work with NCAR's Mesoscale and Microscale Meteorology (MMM) Division and Florida State University (FSU) scientists on the development of these assimilation techniques.

For assimilation of the limb sounding data into atmospheric models the error estimates are as important as the observations. Significant effort will have to be invested by the CDAAC team to determine the full error covariance of the COSMIC profile observations.

\subsubsection{Analysis of open loop tracking in the lower troposphere}

For meteorology it is of paramount importance to collect occultation soundings all the way to the surface. During GPS/MET most soundings did not penetrate to the lowest $\mathrm{km}$ of the atmosphere. For example, during the time period in October 1995 (Rocken et al., 1997) only about $10 \%$ of all soundings reached the lowest $\mathrm{km}$. However, during an earlier time period in June 1995 almost $45 \%$ percent of the occultations reached the bottom $1 \mathrm{~km}$ of the atmosphere. The difference can be attributed to the different receiver tracking firmware versions that had been uploaded to the orbiting receiver. Figure 12 shows the penetration depth between $(0-10$ $\mathrm{km}$ ) of all GPS/MET soundings that have been processed. Note that only very few soundings reach the surface in tropical regions.

The goal for COSMIC is to track $90 \%$ of all rising and setting occultation soundings into the lowest $1 \mathrm{~km}$ of the atmosphere. This will be achieved with (a) at least $10 \mathrm{~dB}$ higher gain antennae and (b) with "open loop" tracking in the lower part of the troposphere. While phase locked loops of ten cannot track the rapidly fluctuating signal in the lowest troposphere, open loop tracking is capable of tracking the phase and amplitude of multiple tones (due to atmospheric multipath) independent of signal dynamics. Half of all profiles will be from rising occultations. All rising occultations will start with open loop tracking data before the receiver can lock its loop several $\mathrm{km}$ above the ground. CDAAC scientists are developing sof tware to reconstruct the open loop data collected on orbit, and investigating open loop sampling rate and Doppler model requirements to allow reliable soundings to near the surface (Sokolovskiy, 2000a). 


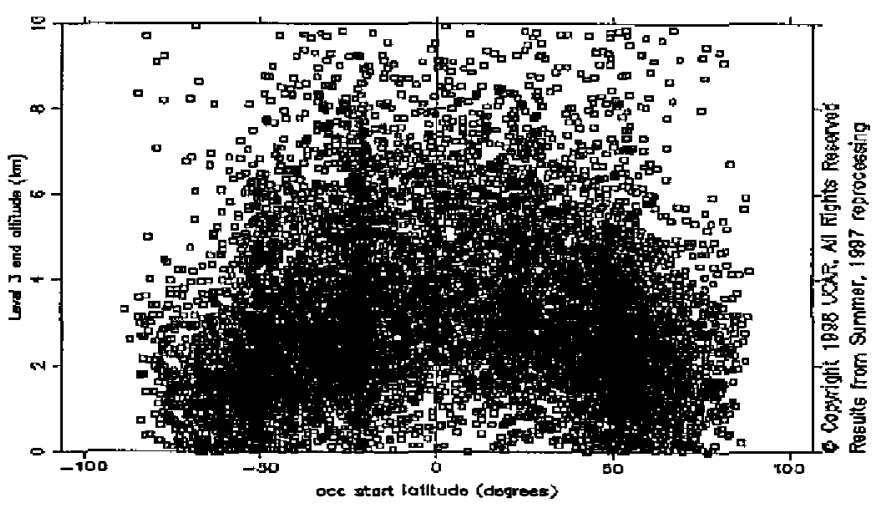

Fig. 12. Penetration depth of GPS/ MET project soundings.

\subsubsection{Fiducial Network Studies}

During GPS/MET we observed high frequency Doppler phase errors, often as high as 1 $\mathrm{mm} / \mathrm{s}$ and in extreme cases $5 \mathrm{~mm} / \mathrm{s}$ (Fig. 7). As evidenced by daily repeatability with the wellknown 4-minute sidereal day shift, these errors were caused by multipath reflections at the ground fiducial sites. Since these errors are large, compared to the required $0.1 \mathrm{~mm} / \mathrm{s}$ satellite velocity errors, we will develop algorithms to reduce this effect. For this task we will explore the use of ground-site multipath correction maps, multipath modeling, and filtering algorithms. COSMIC also needs to collaborate with the operators of the fiducial network to encourage installation of low multipath fiducial sites for LEO mission support.

\subsubsection{Ionospheric Analysis}

The assumption of spherical symmetry along the signal ray, as required by the Abel inversion technique, is of ten invalid because of the very long, several $1000-\mathrm{km}$ path lengths in the ionosphere. A constrained inversion technique (Hajj et al., 1994) can be applied to avoid this assumption. Schreiner et al. $(1998,1999)$ show that this technique, in order to improve upon the Abel inversion, requires a good first guess of the ionospheric electron density gradients along the path transected by the occulting signal. Either TIP measurements, or tomographic solutions of the ionosphere, or both can provide this a priori information for COSMIC. Thus algorithms will have to be developed and implemented to combine GPS occultation and TIP observations. Tomographic solutions will ingest GPS TEC data from LEO, TBB, ground based GPS TEC, and TIP data to produce estimates of 4 dimensional electron density fields, and of GPS ransmitter and receiver inter channel L1/L2 biases. We plan to implement these analysis techniques at the CDAAC, using algorithms developed by ourselves and by our collaborators at NRL, at the University of Texas Applied Research Laboratory (ARL) and by the recently funded Global Assimilation of Ionospheric Measurements (GAIM) team.

\subsubsection{Ionospheric Scintillation}

Scintillation parameters will be computed on orbit from GPS SNR data, and will be forwarded to the TBB ground receiver sites within $\sim 10 \mathrm{~min}$ of the event (Fig. 9). Additional 
scintillation data will be available from ground-based GPS and TBB receivers. These data will reveal the level of scintillation on the various transmission links, but not where the scintillation is occurring along the transmitter-receiver link. In the case when the region of the ionosphere containing irregularities is transected from several directions it is possible to locate a region causing scintillations through tomographic analysis. It is also possible to locate the region containing irregularities along the line of site without multiple transecting rays. Two methods for locating scintillation-causing irregularities will be developed both requiring phase and amplitude of the received signals and different techniques of back propagation. Note that these localization techniques will not be applicable within 10 minutes of the event because of the need for phase and amplitude data, which are not transmitted via the TBB modulation. The CDAAC team will provide a database for the scientific investigation of irregularities of electron density in the ionosphere. Other groups will generate operational communication outage maps using the CDAAC scintillation products.

\subsubsection{Software Development}

We anticipate that about 60,000 lines of code will have to be written, debugged, and tested for a highly accurate, automated, and reliable processing center. The main components of this system will be related to the overall architecture, instrument state-of-health monitoring, data reformatting, orbit analysis, science data inversion, database and archive system, web interface and data distribution. The CDAAC team will develop the software under the Unix Concurrent Version System (CVS). Rigorous linking of source code versions and input parameter file configuration to the scientific output will have to be implemented, as processing techniques will clearly evolve over the duration of the COSMIC mission. Both the CVS and the version tracking were implemented and applied during GPS/MET and will be refined for COSMIC.

We plan to develop detailed documentation in HTML format. This documentation will be required for the CDAAC team and shall also be used for training and instruction.

\subsubsection{Software Tests: Real Data and Data Simulations}

The CDAAC inversion software will be tested with occultation data from other satellite missions. We will test archived GPS/MET data, but also plan to use data from the missions Orsted, SUNSAT, SAC-C and CHAMP. These missions will be flown prior to COSMIC. Especially CHAMP is an important data set for us to use to develop processing software for open loop tracking data in the lower troposphere, and to test new algorithms for improved diffraction and multipath correction. Thus, during the COSMIC development phase, we plan to conduct scientific data analysis from several other campaigns. The results obtained from this analysis will be made available to interested science investigators, and compared with other groups.

The CDAAC team has installed a sophisticated data simulator for two main functions. Simulated data will be used to test parts of the CDAAC system prior to launch to ensure reliability and processing speed. We will simulate observations from the 8-satellite constellation, using NCEP or ECMWF 4-dimensional weather analysis output, and realistic ionospheric 

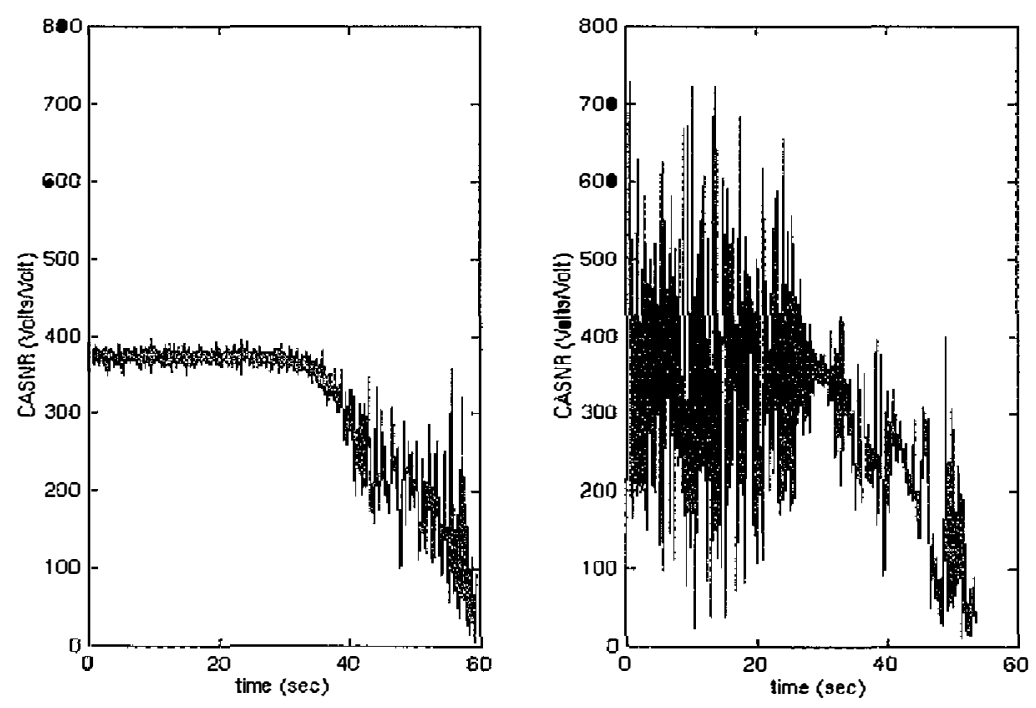

Fig. 13. $50 \mathrm{~Hz}$ GPS C/A SNR data for neutral atmospheric profiles with and without evidence of scintillation. The left panel shows the SNR data for occultation \#0070 at a latitude of $\sim 36$ degrees north and a local time of approximately 22.5 hours. The computed S4 index over the first $20 \mathrm{sec}$ onds of data is 0.005 . The right panel shows evidence of scintillation for occultation \#0077 that occurred at latitude 10 degrees South at a local time of near 23.5 hours. The computed S4 index for occultation \# 0077 over the first 20 seconds of data is 0.113 . The drop in signal strength at -35 seconds in both panels is caused primarily by defocusing as the signal enters the neutral atmosphere.

models. These simulations will help test improvements in the inversion software. We plan to use the ESA-developed EGOPS data simulator, which has been made available including source code to the CDAAC team for collaborative research with our colleagues at the University of Graz, Austria.

\subsubsection{Data Validation}

CDAAC will compute two solutions, one in real-time and one post processed. These solutions will undergo different data validation procedures. The real-time solutions will undergo internal quality checks and will be compared to global 12-hour forecasts. Internal quality checks will be based on signal to noise ratio, phase breaks (cycle slips), signal bending characteristics in the "zero bending" $60-80 \mathrm{~km}$ height region, ionospheric activity, and accumulated empirical error statistics.

Focussing and defocusing of the limb-sounding signal as it descends through the atmosphere causes amplitude fluctuations. During GPS/MET we developed algorithms to invert amplitude data into temperature and refractivity profiles (Sokolovskiy, 2000b). These ampli- 
tude-derived profiles can be used for validating the phase derived profiles to detect large errors.

Post processed results will undergo the same tests plus much more extensive comparisons with correlative data, from radiosondes, ground-based GPS, Lidars, and space-based systems such as TOVS GOES, AIRS/AMSU. An important task during the CDAAC development phase will be to establish the automated processes that collect the correlative data, populate the correlative database, and carry out the comparisons by applying the appropriate interpolations in space and time.

Occultation analysis centers at JPL, the University of Arizona, in Europe, and elsewhere will also process COSMIC soundings. We plan to establish data comparison procedures with these centers.

\subsubsection{Setup of CDAAC hardware}

The development tasks described above will be carried out on a smaller CDAAC development system because we want to purchase the main analysis system as close to the launch date as possible. We plan to execute the purchase and begin setup and networking about 9 months prior to launch. This will allow for sufficient time to install the software, test it on the operational system, test all connections to the data sources, test collection of correlative data, and test the data distribution system. Our goal is to be ready to process all data immediately after launch.

The GPS/MET experience has taught us that this is very important. Only after the end of the GPS/MET mission did we notice that the JPL-developed firmware, which had been uploaded during the early June-July, 1995 prime time period, allowed more reliable tropospheric penetration than the firmware that was used for the remaining 2 years of the mission. Had we been able to process, and validate large amounts of data in near real time during the early part of the mission, we would have obtained significantly more lower-tropospheric observations from GPS/MET.

\subsubsection{Operating CDAAC}

Almost immediately upon launch the data from the satellites and the ground support network will begin to flow to the CDAAC. Depending on the constellation design, it may take as long as 9-months for the satellites to reach their final orbits and full global coverage. During this transition phase GPS data will already be processed for gravity field improvement, satellite cross-checks, and for special occultation studies. While the satellites remain closely clustered, 4000 daily occultations will occur over smaller, localized regions, affording a unique opportunity for data validation field campaigns, special tomographic studies, and experiments to resolve fine scale atmospheric features. Transition phase and operational phase data will all be analyzed in the same way, and the same main levels of products will be made available.

Acknowledgments Development of the COSMIC System was supported by the National Space Program Office (NSPO) in Taiwan. The National Science Foundation (NSF) the National Oceanographic and Atmospheric Administration (NOAA) and the National Aeronau- 
tics and Space Administration (NASA) supported the GPS/MET project and the development of the COSMIC Data Analysis and Archive Center (CDAAC). Development of the TIP and Beacon instruments is supported by the Office for Naval Research (ONR). Development of the GPS instrument for GPS/MET and for COSMIC is supported by the National Aeronautics and Space Administration (NASA).

\section{REFERENCES}

Anthes, R., M. Exner, C. Rocken, and R. Ware, 1997: Results from the GPS/MET Experiment and Potential Applications to GEWEX. GEWEX News, 7, 3-6.

Bernhardt et al., 1998: Two-dimensional mapping of the plasma density in the upper atmosphere with computerized ionospheric tomography (CIT). Phys. of Plasmas, 5, 20102021.

Bust, G. S., J. A. Cook, G. R. Kronschnabl, C. J. Vasicek, and S. B. Ward, 1994: Int. J. Imag. Sys. Tech, 5, 160-168.

Crook, N. A., 1996: Sensitivity of Moist Convection Forced by Boundary Layer Processes to Low-Level Thermodynamic Fields. Mon. Weather Rev., 124, 1767.

Eyre, J., 1994: Assimilation of radio occultation measurements into a numerical weather prediction system, Tech. Memo.199, Eur. Cent. For Medium Range Weather Forecasts, Reading, England.

Feng, D., B. Herman, M. Exner, B. Schreiner, R. Anthes, and R. Ware, 1995: Space-borne GPS Remote Sensing for Atmospheric Research, Proceedings from Synthetic Aperture Radar and Passive Microwave Sensing, 2584, 448-455.

Gorbunov, M. E., and A. S. Gurvich, 1998: Microlab-1 experiment: Multipath effects in the lower troposphere. J. Geophys. Res, 103, 13819-13826.

Gorbunov, M. E., A. S. Gurvich, and L. Bengtsson, 1996: Advanced algorithms of inversion of GPS/MET satellite data and their applications to reconstruction of temperature and humidity, Rep. 211, Max Planck Inst. For Meteorology, Hamburg.

Gurvich, A. S., and S. V. Sokolovskiy, 1983: Reconstruction of a pressure field by remote refractometry from space, Izvestya. Atm. and Ocean. Phys., 21, 7-13.

Hajj, G. A., R. Ibanez-Meir, E. R. Kursinski, L. J. Romans, 1994: Imaging the Ionosphere with the GPS. Inter. J. Imaging Systems and Tech., 5, 174-184.

Hajj, G. A. and L. J. Romans, 1998: Ionospheric electron density profiles obtained with the GPS: Results from the GPS/MET experiment, Radio Science, Vol. 33, No. 1, pp.175190, Jan-Feb.

Hocke K., 1997: Inversion of GPS meteorology data. Ann. Geophys., 15, 443-450.

Hoeg, P., A. Hauchecorne, G. Kirchengast, S. Syndergaard, B. Belloul, R. Leitinger, and W. Rothleitner, 1996: Derivation of atmospheric properties using a radio occultation technique. Sci. Rep., 95-4, Danish Meteorol. Inst..

Howe, B. M., K. Runciman, J. A. Secan, 1998: Tomography of the ionosphere: Four-dimensional simulations. Radio Science, Vol. 33, pp.109-128.

Kuo, Y.-H., X. Zou, and W. Huang, 1997: The Impact of GPS Data on the Prediction of an 
Extratropical Cyclone: An Observing System Simulation Experiment. J. Dyn. Atmos. Ocean, 27, 439-470.

Kuo, Y.-H., X. Zou, S.-J. Chen, Y.-R. Guo, W. Huang, R. Anthes, D. Hunt, M. Exner, C. Rocken, S. Sokolovskiy, 1998: A GPS/MET sounding through an intense upper-level front. Bull. Am. Meteorol. Soc., 79, 617-626.

Karayel, E.T. and D. P. Hinson, 1997: Sub-Fresnel vertical resolution in atmospheric profiles from radio occultation. Radio Sci. 32, 411-423.

Kursinski, E. R., G. A. Hajj, W. I. Bertiger, S. S. Leroy, T. K. Meehan, L. J. Romans, J. T. Schofield, D. J. McCleese, W. G. Melbourne, C. L. Thournton, T. P. Yunck, J. R. Eyre, R. N. Nagatani, 1996: Initial Results of Radio Occultation Observations of Earth's Atmosphere Using the Global Positioning System. Science, 271, 1107-1110.

Kursinski, E.R., G.A. Hajj, J.T. Schofield, R.P. Linfield and K.R. Hardy, 1997: Observing the Earth's atmosphere with radio occultation measurements using the Global Positioning System, J. Geophys. Res, Vol. 102, NO. D19, pp.23429-23465, Oct. 20.

Lemoine, F.G., et al., 1998: The Development of the Joint NASA GSFC and the National Imagery and Mapping Agency (NIMA) Geopotential ModeI EGM96, NASA/TP-1998206862.

Leroy, S. S., 1997: The measurement of geopotential heights by GPS radio occultation. $J$. Geophys. Res., 102, 6971-6986.

Leroy, S. S., 1998: Optimal detection of global warming using temperature profiles: A methodology. J. Climate, in press.

Lindzen, R. S., 1990: Some coolness concerning global warming. Bull. Am. Meteorl. Soc. 71, 288.

Melbourne, W., E. Davis, C. Duncan, G. Hajj, K. Hardy, E. Kursinski, T. Meehan, L. Young, and T. Yunck, 1994: The application of space borne GPS to atmospheric limb sounding and global change monitoring. JPL Publ., 94-18, 147 pp..

North, G. R., and M. J. Stevens, 1998: Detecting climate signals in the surface temperature record. J. Climate, 11, 563-577.

Richmond, A. D., and Y. Kamide, 1988: Mapping electrodynamic features of the high-latitude ionosphere from localized observations: Technique. J. Geophys. Res., 93, 57415759.

Rind, D., 1998: Just add water vapor. Science, 281, 1152.

Rius, A., G. Ruffini, and L. Cucurull, 1997: Improving the vertical resolution of ionospheric tomography with GPS occultations. Geophysical Research Letters, Vol. 24, No. 18, pp. 2291-2294, Sep..

Rius, A., G. Ruffini, and A. Romeo, 1998: Analysis of Ionospheric Electron Density Distribution from GPS/MET Occultations. IEEE Trans. on Geoscience and Remote Sensing, Vol. 36, No. 2, Mar..

Rocken, C., R. Anthes, M. Exner, D. Hunt, S. Sokolovskiy, R. Ware, M. Gorbunov, W. Schreiner, D. Feng, B. Herman, Y. Kuo, and X. Zou, 1997: Analysis and validation of GPS/MET data in the neutral atmosphere. J. Geophys. Res., 102, 29849-29866.

Schreiner, W. S., D. C. Hunt, C. Rocken, S. Sokolovskiy, 1998: Precise GPS data processing for the GPS/MET radio occultation mission at UCAR, Proc. of the Institute of Naviga- 
tion- Navigation 2000, Long Beach, CA, pp.103-112, Jan 21-23.

Schreiner, W.S., S. Sokolovskiy, C. Rocken, and D. C. Hunt, 1999: Analysis and Validation of GPS/MET Radio Occultation Data in the Ionosphere. Radio Science, Vol. 34, No. 41, pp.949-966, July-August.

Schutz, B., P. Abusali, C. Schroeder, B. Tapley, M. Exner, R. McCloskey, R. Carpenter, M. Cooke, S. McDonald, N. Combs, C. Duncan, C. Dunn, and T. Meehan, 1995: GPS Tracking Experiment of a Free-Flyer Deployed From Space Shuttle. Proceedings of GPS-95, Institute of Navigation.

Solheim, J. Vivekanandan, F., R. Ware, and C. Rocken, 1999: Propagation delays induced in GPS signals by dry air, water vapor, hydrometeors, and other particulates. J. Geophys. Res., 104, No. D8, pp.9663-9670, April 27.

Sokolovskiy S., 2000a: Signal Dynamics and Measurement of Atmospheric Limb Sounding Signals in the lower Troposphere. Submitted to Radio Science.

Sokolovskiy S., 2000b: Inversions of Radio-Occultation Amplitude Data. Radio Science, (in press).

Space Handbook, editors C. D. Cochran, D. M. Gorman and J. D. Doumoulin, 1985: Air University Press, Maxwell AFB, Ala., January.

Stevens, M. J., 1999: Optimal climate signal detection in four dimensions. J. Geophys. Res. Atmos. 104, 4089-4099.

Tsuda, T., Nishida N., Rocken C., R. Ware, 2000: A global morphology of gravity wave activity in the stratosphere revealed by the GPS occultation data (GPS/MET). in print JGR.

Voro'bev, V. V., and T. G. Krasil'nikova, 1994: Estimation of the Accuracy of the Atmospheric Refractive Index Recovery from Doppler Shift Measurements at Frequencies used in the NAVSTAR System. Phys. Atmos. Oceans, 29, 602-609.

Ware, R., M. Exner, D. Feng, M. Gorbunov, K. Hardy, B. Herman, Y. Kuo, T. Meehan, W. Melbourne, C. Rocken, W. Schreiner, S. Sokolovskiy, F. Solheim, X. Zou, R. Anthes, S. Businger, and K. Trenberth, 1996: GPS Sounding of the Atmosphere From Low Earth Orbit: Preliminary Results. Bull. Amer. Meteor. Soc., 77, 19-40.

Ware, R. H., C. Alber, C. Rocken, F. Solheim, Sensing integrated water vapor along GPS ray paths, Geophys. Res. Lett., Vol. 24, No. 4, pp 417-420, Feb. 15, 1997.

Yuan, L., R. A. Anthes, R. H. Ware, C. Rocken, W. Bonner, M. Bevis, and S. Businger, 1993: Sensing Climate Change Using the Global Positioning System. J. Geophys. Res., 98, $14,925-14,937$.

Zou, X. , Y.-H. Kuo, and Y.-R. Guo, 1995: Assimilation of Atmospheric Radio Refractivity Using a Nonhydrostatic Mesoscale Model. Mon. Wea. Rev., 123, 2229-2249.

Zou, X., F. Vandenberghe, B. Wang, M. E. Gorbunov, Y.-H. Kuo, S. Sokolovskiy, J. C. Chang, J. G. Sela, and R. A. Anthes, 1999: A ray-tracing operator and its adjoint for the use of GPS/MET refraction angle measurements. J. Geophys. Res.-Atmosphere, 104, NO. D18, 22301-22318.

Zou, X., B. Wang, H. Liu, R. A. Anthes, T. Matsumura, and Y.-J. Zhu, 2000: Use of GPS/ MET refraction angles in 3D variational analysis. Q. J. R. Met. Soc., (in press). 